\title{
Transmit Precoding and Receive Power Splitting for Harvested Power Maximization in MIMO SWIPT Systems
}

Deepak Mishra and George C. Alexandropoulos

The self-archived postprint version of this journal article is available at Linköping University Institutional Repository (DiVA):

http:/ / urn.kb.se/ resolve?urn=urn:nbn:se:liu:diva-155769

N.B.: When citing this work, cite the original publication.

Mishra, D., Alexandropoulos, G. C., (2018), Transmit Precoding and Receive Power Splitting for Harvested Power Maximization in MIMO SWIPT Systems, IEEE Transactions on Green

Communications and Networking, 2(3), 774-786. https:// doi.org/ 10.1109/TGCN.2018.2835409

Original publication available at:

https:/ / doi.org/ 10.1109/ TGCN.2018.2835409

Copyright: Institute of Electrical and Electronics Engineers (IEEE)

http:// www.ieee.org/index.html

(C)2018 IEEE. Personal use of this material is permitted. However, permission to reprint/republish this material for advertising or promotional purposes or for creating new collective works for resale or redistribution to servers or lists, or to reuse any copyrighted component of this work in other works must be obtained from the IEEE. 


\title{
Transmit Precoding and Receive Power Splitting for Harvested Power Maximization in MIMO SWIPT Systems
}

\author{
Deepak Mishra, Member, IEEE and George C. Alexandropoulos, Senior Member, IEEE
}

\begin{abstract}
We consider the problem of maximizing the harvested power in Multiple Input Multiple Output (MIMO) Simultaneous Wireless Information and Power Transfer (SWIPT) systems with power splitting reception. Different from recently proposed designs, with our optimization problem formulation we target for the jointly optimal transmit precoding and receive uniform power splitting (UPS) ratio maximizing the harvested power, while ensuring that the quality-of-service requirement of the MIMO link is satisfied. We assume practical RadioFrequency (RF) energy harvesting $(\mathrm{EH})$ receive operation that results in a non-convex optimization problem for the design parameters, which we first formulate in an equivalent generalized convex problem that we then solve optimally. We also derive the globally optimal transmit precoding design for ideal reception. Furthermore, we present analytical bounds for the key variables of both considered problems along with tight high signal-to-noise ratio approximations for their optimal solutions. Two algorithms for the efficient computation of the globally optimal designs are outlined. The first requires solving a small number of nonlinear equations, while the second is based on a two-dimensional search having linear complexity. Computer simulation results are presented validating the proposed analysis, providing key insights on various system parameters, and investigating the achievable EH gains over benchmark schemes.
\end{abstract}

Index Terms-RF energy harvesting, MIMO, precoding, power splitting, rate-energy trade off, SWIPT, nonconvex optimization.

\section{INTRODUCTION}

There has been recently increasing interest [2]-[4] in utilizing Radio Frequency (RF) signals for transferring simultaneously energy and data, also known as Simultaneous Wireless Information and Power Transfer (SWIPT). This technology can play a major role in the practical ubiquitous deployment of low power wireless devices in fifth generation (5G) wireless networks and beyond [4]-[7]. Particularly, it can be one of the promising candidates for enabling the perpetual operation of small cells, Internet-of-Things (IoT) [2], Machine-to-Machine (M2M) communications and cognitive radio networks [5]-[7].

Despite these merits, SWIPT suffers from some fundamental bottlenecks. First and foremost, the signal processing

D. Mishra was the Department of Electrical Engineering, Indian Institute of Technology Delhi, 110016 New Delhi, India. Now he is with the Department of Electrical Engineering, Linköping University, 58183 Linköping, Sweden (e-mail: deepak.mishra@liu.se).

G. C. Alexandropoulos is with the Mathematical and Algorithmic Sciences Lab, France Research Center, Huawei Technologies France SASU, 92100 Boulogne-Billancourt, France (e-mail: george.alexandropoulos@ huawei.com).

A preliminary conference version [1] of this work was presented at the IEEE CAMSAP, Curaçao, Dutch Antilles, Dec. 2017. and resource allocation strategies for wireless information and energy transfer differ significantly for achieving their respective goals [8], [9]. In fact, there exists a non-trivial trade off between information and energy transfer that necessitates thorough investigation for optimizing the SWIPT performance. In addition, this performance is impacted by the low energy sensitivity and RF-to-Direct Current (DC) rectification efficiency [3]. Another practical concern is that the existing RF EH circuits cannot decode the information directly and vice-versa [10], [11]. Lastly, the available solutions [12], [13] for realizing practical SWIPT gains require high complexity and are still far from providing analytical insights. To confront with these bottlenecks, Multiple-Input-MultipleOutput (MIMO) technology and resource allocation schemes as well as cooperative relaying strategies have been recently considered [3], [10]-[22]. In this paper, we are interested in optimizing the efficacy of MIMO systems for efficient SWIPT.

\section{A. State-of-the-Art}

The non-trivial trade off between information capacity and average received power was firstly investigated in [8], [9] for a Single-Input-Single-Output (SISO) link. Then, the authors in [11] discussed why the SWIPT theoretical gains are difficult to realize in practice and proposed some practical Receiver (RX) architectures. Among them belong the Time Switching (TS), Power Splitting (PS), and Antenna Switching (AS) [14] architectures that use one portion of the received signal (in time, power, or space) for EH and another one for Information Decoding (ID). In [12], Transmitter (TX) precoding techniques for efficient MIMO SWIPT systems were presented. Recently, Spatial Switching (SS) was proposed [16] that first decomposes MIMO channel to its spatial eigenchannels and then assigns some for energy and some for information transfer [10].

The aforementioned SWIPT RX architectures have been lately considered in various MIMO systems [16]-[22]. For example, the transmit power minimization satisfying both energy and rate requirements was investigated in [16] for MIMO SWIPT with SS. In [17], a Semi-Definite Programming (SDP) relaxation technique for a multi-user multiple-input single-output system was used to study the joint TX precoding and PS optimization. A second-order cone programming relaxation solution for the latter problem with significantly reduced computational complexity than SDP was proposed in [18]. In [19] and [20], more general MIMO interference channels were investigated adopting the interference alignment 
technique. Authors in [21] considered a multi-antenna full duplex access point and a single-antenna full duplex user, and investigated the joint design of TX precoding and RX PS ratio for minimizing the weighted sum transmit power. However, these MIMO SWIPT works presented suboptimal iterative algorithms based on convex relaxation which that are unable to provide key insights on the joint optimal design.

\section{B. Motivation and Key Contributions}

A major goal of RF EH systems is the optimization of the end-to-end EH efficiency [2] by maximizing the rateconstrained harvested energy for a given TX power budget. This is in principle challenging with the available EH circuitry implementations, where the RF-to-DC rectification is a nonlinear function of the received RF power [22]-[25]. This fact leads naturally to the necessity of optimizing the harvested power rather than the receiver power treated in the existing literature [12]-[21]; therein, constant RF-to-DC rectification efficiency has been assumed. In this paper, we study the problem of maximizing the harvested power in MIMO SWIPT systems with practical PS reception [12], while ensuring that the quality-of-service requirement of the MIMO link is met. We note that, although the PS architecture involves higher RX complexity, it is more efficient than TS since the received signal is used for both EH and ID. In addition, PS is more suitable for delay-constraint applications. We are interested in finding the jointly optimal TX precoding scheme and the RX Uniform PS (UPS) ratio for the considered optimization problem, and in gaining analytical insights on the interplay among various system parameters. To our best of knowledge, this joint optimization problem for maximizing the harvested DC power has not been considered in the past, and available designs for practical MIMO SWIPT are suboptimal. The key contributions of this paper are summarized below.

- We present an equivalent generalized convex formulation for the considered non-convex harvested power maximization problem that helps us in deriving the global jointly optimal TX precoding and RX UPS ratio design. We also present the globally optimal TX precoding design for ideal reception. For both designs there exists a rate requirement value determining whether the TX precoding operation is energy beamforming or information spatial multiplexing. This novel feature stems from our novel formulation involving rate constrained $\mathrm{EH}$ optimization and does not appear in available designs [12], [16]-[22].

- We investigate the trade off between the harvested power and achievable information rate for both globally optimal designs. Practically motivated asymptotic analysis for obtaining computationally efficient optimal solution in the high Signal-to-Noise-Ratio (SNR) regime is provided.

- We detail a computationally efficient algorithm for the global optimal design and present a low complexity alternative algorithm based on a two-dimensional (2-D) linear search. The complexity of the latter algorithm is linear in the number of MIMO spatial eigenchannels.

- We carry out a detailed numerical investigation of the presented joint optimal solutions to provide insights on

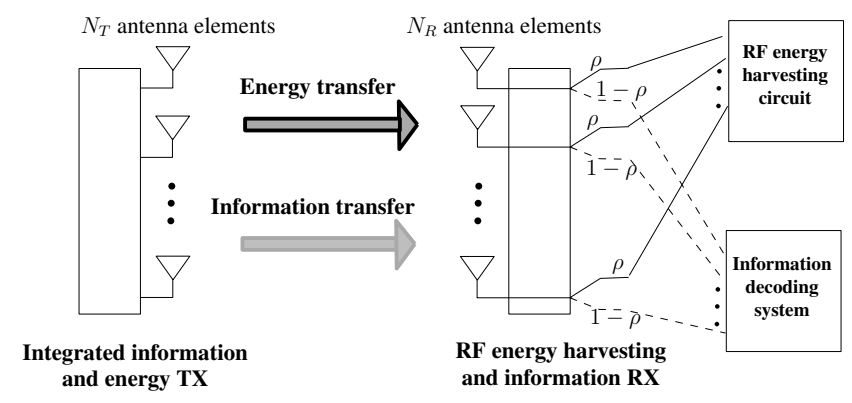

Fig. 1. Adopted MIMO SWIPT system model with UPS $\rho$ reception.

the impact of key system parameters on the trade off between harvested power and achievable information rate.

The key challenges with our problem formulation addressed in this paper include its generalized convexity proof given the non-linear rectification property and the analytical exploration of non-trivial insights on its controlling variables, which helped us in designing a low complexity global optimization algorithm. Additionally, we would like to emphasize that our performance results are valid for any practical RF EH circuit model [22]-[24], and our key system design insights can be extended to investigate multiuser MIMO SWIPT systems.

Notations: Vectors and matrices are denoted by boldface lowercase and boldface capital letters, respectively. The transpose and Hermitian transpose of $\mathbf{A}$ are denoted by $\mathbf{A}^{\mathrm{T}}$ and $\mathbf{A}^{\mathrm{H}}$, respectively, and $\operatorname{det}(\mathbf{A})$ is the determinant of $\mathbf{A}$, while $\mathbf{I}_{n}(n \geq 2)$ is the $n \times n$ identity matrix and $\mathbf{0}_{n}(n \geq 2)$ is the $n$-element zero vector. The trace of $\mathbf{A}$ is denoted by $\operatorname{tr}(\mathbf{A})$, $[\mathbf{A}]_{i, j}$ stands for $\mathbf{A}$ 's $(i, j)$-th element, $\lambda_{\max }(\mathbf{A})$ represents the largest eigenvalue of $\mathbf{A}$, and $\operatorname{diag}\{\cdot\}$ denotes a square diagonal matrix with a's elements in its main diagonal. $\mathbf{A} \succeq 0$ and $\mathbf{A} \succ 0$ mean that $\mathbf{A}$ is positive semi definite and positive definite, respectively. $\mathbb{C}$ represents the complex number set, $(x)^{+} \triangleq \max \{0, x\},\lceil x\rceil$ denotes the smallest integer larger than or equal to $x, \mathbb{E}\{\cdot\}$ denotes the expectation operator, and $\mathcal{O}(\cdot)$ is the Big $\mathrm{O}$ notation denoting order of complexity.

\section{SyStem AND CHANNEL MODELS}

We consider the MIMO SWIPT system of Fig. 1, where the TX is equipped with $N_{T}$ antenna elements and wishes to simultaneously transmit information and energy to the RFpowered RX having $N_{R}$ antenna elements. We assume a frequency flat MIMO fading channel $\mathbf{H} \in \mathbb{C}^{N_{R} \times N_{T}}$ that remains constant during one transmission time slot and changes independently from one slot to the next. The channel is assumed to be perfectly known at both TX and RX. The entries of $\mathbf{H}$ are assumed to include independent, zero-mean circularly symmetric complex Gaussian (ZMCSCG) random variables with unit variance. So, the rank of $\mathbf{H}$ is $r=\min \left(N_{R}, N_{T}\right)$. The baseband received signal $\mathbf{y} \in \mathbb{C}^{N_{R} \times 1}$ at $\mathrm{RX}$ is given by

$$
\mathbf{y}=\mathbf{H x}+\mathbf{n}
$$

where $\mathbf{x} \in \mathbb{C}^{N_{T} \times 1}$ denotes the transmitted signal with covariance matrix $\mathbf{S} \triangleq \mathbb{E}\left\{\mathbf{x x}^{\mathrm{H}}\right\}$ and $\mathbf{n} \in \mathbb{C}^{N_{R} \times 1}$ represents the AWGN vector having ZMCSCG entries each with variance $\sigma^{2}$. The elements of $\mathbf{x}$ are assumed to be statistically independent, 


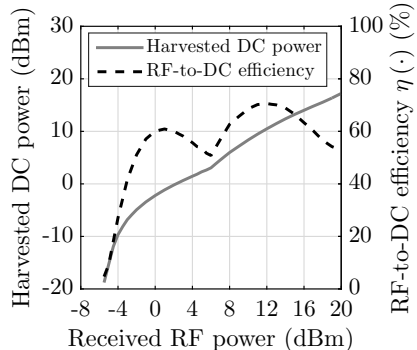

(a) P1110 EVB characteristics [24].

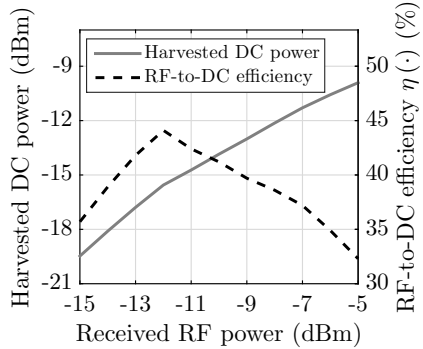

(b) RF EH circuit designed in [26].
Fig. 2. Variation of harvested DC power and RF-to-DC efficiency with received RF power for practical two EH circuits [24], [26].

the same is assumed for the elements of $\mathbf{n}$. For the transmitted signal we finally assume that there exists an average power constraint across all TX antennas denoted by $\operatorname{tr}(\mathbf{S}) \leq P_{T}$.

Capitalizing on the signal model in (1), the average received power $P_{R}$ across all RX antennas can be obtained as $P_{R} \triangleq \mathbb{E}\left\{\mathbf{y}^{\mathrm{H}} \mathbf{y}\right\}$. Note that the averaging is performed over the transmitted symbols during each coherent channel block. As the noise strength (generally lower than $-80 \mathrm{dBm}$ ) is much below than the received energy sensitivity of practical RF EH circuits (which is around $-20 \mathrm{dBm}$ ) [2], we next neglect the contribution of $\mathbf{n}$ to the harvested power. Note, however, that the analysis and optimization results of this paper can be easily extended for non-negligible noise power scenarios. We therefore rewrite $P_{R}$ as the following function of $\mathbf{H}$ and $\mathbf{x}$

$$
P_{R} \triangleq \mathbb{E}\left\{\mathbf{x}^{\mathrm{H}} \mathbf{H}^{\mathrm{H}} \mathbf{H} \mathbf{x}\right\}=\operatorname{tr}\left(\mathbf{H} \mathbf{S} \mathbf{H}^{\mathrm{H}}\right)
$$

As demonstrated in Fig. 1, we consider UPS ratio $\rho \in[0,1]$ at each RX antenna element. This ratio reveals that $\rho$ fraction of the received signal power at each antenna is used for RF $\mathrm{EH}$, while the remaining $1-\rho$ fraction is used for ID. With this setting together with the previous noise assumption, the average total received power $P_{R, E}$ available for RF EH is given by $P_{R, E} \triangleq \rho P_{R}=\rho \operatorname{tr}\left(\mathbf{H S H}^{\mathrm{H}}\right)$. This definition for the average received power is the most widely used definition [12], [13] for investigating the performance lower bound with the PS RX architectures. Supposing that $\eta(\cdot)$ denotes the RFto-DC rectification efficiency function, which is in general a non-linear positive function of the received RF power $P_{R, E}$ available for EH [22]-[24], the total harvested DC power is obtained as $P_{H} \triangleq \eta\left(\rho P_{R}\right) \rho P_{R}$. Despite this circuit dependent non-linear relationship between $\eta$ and $P_{R, E}$, we note that $P_{H}$ is monotonically non-decreasing in $P_{R, E}=\rho P_{R}$ for any practical RF EH circuit [22]-[24] due to the law of energy conservation. For instance, to give more insights, we plot both $\eta$ and $P_{H} \triangleq \eta\left(P_{R, E}\right) P_{R, E}$ as a function of the received RF power $P_{R, E}$ variable at the input of two realworld RF EH circuits, namely, (i) the commercially available Powercast P1110 evaluation board (EVB) [24] and (ii) the circuit designed in [26] for low power far field RF EH in Figs. 2(a) and 2(b), respectively. So, using $P_{H}=\mathcal{F}\left(P_{R, E}\right)$, where $\mathcal{F}(\cdot)$ represents a non-linear non-decreasing function, we are able to obtain the jointly global optimal design.

\section{JOINT TX AND RX OPTIMIZATION FRAMEWORK}

Here we present the mathematical formulation of the optimization problem. Then in Section III-A, we consider the practical case of UPS reception and prove an interesting property of the underlying optimization problem that will be further exploited in Section IV for deriving the globally optimal design. Aiming at comparing with the ideal reception case, we present its mathematical formulation in Section III-B.

\section{A. UPS Reception}

Focusing on the MIMO SWIPT model of Section II, we consider the problem of designing the covariance matrix $\mathbf{S}$ at the multi-antenna TX and UPS ratio $\rho$ at the multi-antenna EH RX for maximizing the total harvested DC power, while satisfying a minimum instantaneous rate requirement $R$ in bits per second (bps) per $\mathrm{Hz}$ for information transmission. So, the proposed design framework is mathematically expressed as:

$$
\begin{array}{ll}
\mathcal{O P}: \max _{\rho, \mathbf{S}} \quad P_{H}=\eta\left(\rho \operatorname{tr}\left(\mathbf{H S H} \mathbf{H}^{\mathrm{H}}\right)\right) \rho \operatorname{tr}\left(\mathbf{H S H}^{\mathrm{H}}\right) \\
\text { s.t. } \quad(\mathrm{C} 1): \log _{2}\left(\operatorname{det}\left(\mathbf{I}_{N_{R}}+(1-\rho) \sigma^{-2} \mathbf{H} \mathbf{S} \mathbf{H}^{\mathrm{H}}\right)\right) \geq R, \\
\quad(\mathrm{C} 2): \operatorname{tr}(\mathbf{S}) \leq P_{T},(\mathrm{C} 3): \mathbf{S} \succeq 0,(\mathrm{C} 4): 0 \leq \rho \leq 1,
\end{array}
$$

where constraint $(\mathrm{C} 1)$ represents the minimum instantaneous rate requirement, $(\mathrm{C} 2)$ is the average transmit power constraint, while (C3) and (C4) are the boundary conditions for $\mathrm{S}$ and $\rho$. It can be easily concluded that $P_{H}$ is jointly nonconcave in regards to the unknown variables $\mathbf{S}$ and $\rho$. However, in the following Lemma 1 we show that the received RF power $P_{R, E}$ available for $\mathrm{EH}$ is jointly pseudoconcave in $\mathbf{S}$ and $\rho$.

Lemma 1: $P_{R, E}$ is jointly pseudoconcave in $\mathbf{S}$ and $\rho$.

Proof: With $\operatorname{tr}\left(\mathbf{H S H}^{\mathrm{H}}\right)$ being linear in $\mathbf{S}$, we deduce that the total average received RF power $P_{R, E}=$ $\rho \operatorname{tr}\left(\mathbf{H S H}^{\mathrm{H}}\right)$ available for $\mathrm{EH}$ is the product of two positive linear functions of $\rho$ and $\mathbf{S}$. Since the product of two positive linear (or concave) functions is log-concave [27, Chapter 3.5.2] and a positive log-concave function is also pseudoconcave [13, Lemma 5], $P_{R, E}$ is jointly pseudoconcave in $\mathbf{S}$ and $\rho$. We now show that solving $\mathcal{O P}$ is equivalent to problem $\mathcal{O P} 1$ :

$$
\mathcal{O P} 1: \max _{\rho, \mathbf{S}} P_{R, E}=\rho \operatorname{tr}\left(\mathbf{H S H}^{\mathrm{H}}\right) \text {, s.t. }(\mathrm{C} 1),(\mathrm{C} 2),(\mathrm{C} 3),(\mathrm{C} 4) .
$$

Proposition 1: Solution pair $\left(\mathbf{S}^{*}, \rho^{*}\right)$ of $\mathcal{O P} 1$ solves $\mathcal{O P}$.

Proof: Irrespective of the circuit-dependent non-linear relationship between $\eta$ and $P_{R, E}, P_{H}$ is monotonically nondecreasing in $P_{R, E}$ [22]-[24]. It can be concluded from [27], [28] that the monotonic non-decreasing transformation $P_{H}$ of the pseudoconcave function $P_{R, E}$ is also pseudoconcave and possesses the unique global optimality property [28, Props. 3.8 and 3.27]. This reveals that $\mathcal{O P}$ and $\mathcal{O P} 1$ are equivalent [29], sharing the same solution pair $\left(\mathbf{S}^{*}, \rho^{*}\right)$.

It can be deduced from Proposition 1 that one may solve $\mathcal{O P} 1$ and then use the resulting maximum received power $P_{R, E}^{*}=$ $\rho^{*} \operatorname{tr}\left(\mathbf{H S}^{*} \mathbf{H}^{\mathrm{H}}\right)$ to compute the maximum harvested power as $P_{H}^{*}=\eta\left(P_{R, E}^{*}\right) P_{R, E}^{*}$. Although $\mathcal{O P} 1$ is nonconvex, we prove in the following theorem a specific property for it that will be used in Section IV to derive its optimal solution.

Theorem 1: $\mathcal{O P} 1$ is a generalized convex problem and its optimal solution can be obtained by solving KKT conditions. 
Proof: As shown in Lemma $1, P_{R, E}$ is jointly pseudoconcave in $\mathbf{S}$ and $\rho$. It follows from constraint $(\mathrm{C} 1)$ that the function $R-\log _{2}\left(\operatorname{det}\left(\mathbf{I}_{N_{R}}+(1-\rho) \sigma^{-2} \mathbf{H S H}^{\mathrm{H}}\right)\right)$ is jointly convex on $\rho$ and $\mathbf{S}$; this ensues from the fact that the matrix inside the determinant is a positive definite matrix [12], [17][19]. In addition, constraints (C2) and (C3) are linear with respect to $\mathbf{S}$ and independent of $\rho$, and constraint (C4) depends only on $\rho$ and is convex. The proof completes by combining the latter findings and using them in [29, Theorem 4.3.8].

Capitalizing on the findings of Proposition 1 and Theorem 1, we henceforth focus on the maximization of the received RF power $P_{R, E}$ for EH. The jointly optimal TX precoding and UPS design for this problem will also result in the maximization of the harvested DC power $P_{H}^{*}$ for any practical RF EH circuitry. Also, the proposed joint transceiver design in this paper is different from the ones in existing works [12][21] considering the received RF power for EH as a constraint and using a trivial linear RF EH model for their investigation.

\section{B. Ideal Reception}

To investigate the theoretical upper bound for $P_{R, E}$, we now consider an ideal RX architecture capable of using all received RF power for both EH and ID. In particular, we remove $\rho$ from $\mathcal{O P} 1$ and $(\mathrm{C} 1)$ and consider the following optimization:

$$
\begin{array}{r}
\mathcal{O P} 2: \max _{\mathbf{S}} P_{R}=\operatorname{tr}\left(\mathbf{H S H}^{\mathrm{H}}\right), \quad \text { s.t. } \quad(\mathrm{C} 2), \quad(\mathrm{C} 3), \\
(\mathrm{C} 5): \log _{2}\left(\operatorname{det}\left(\mathbf{I}_{N_{R}}+\sigma^{-2} \mathbf{H} \mathbf{S} \mathbf{H}^{\mathrm{H}}\right)\right) \geq R .
\end{array}
$$

From the findings in the proof of Theorem 1, the objective function $P_{R}$ of $\mathcal{O P} 2$ along with constraints (C2) and (C3) are linear in $\mathbf{S}$. In addition, (C5) is convex due to the concavity of logarithm with respect to $\mathbf{S}$. Combining the latter facts yields that $\mathcal{O P} 2$ is a convex problem, and hence, its optimal solution can be found using the Lagrangian dual method [27], [29].

\section{Optimal TX Precoding And RX Power Splitting}

Here, we first investigate trade off between energy beamforming and information spatial multiplexing in $\mathcal{O P} 1$. Then, we present the global optimal solutions for $\mathcal{O P} 1$ and $\mathcal{O P} 2$.

\section{A. Energy Beamforming versus Spatial Multiplexing}

Let us consider the reduced Singular Value Decomposition (SVD) of the MIMO channel matrix $\mathbf{H}=\mathbf{U} \boldsymbol{\Lambda} \mathbf{V}^{\mathrm{H}}$, where $\mathbf{V} \in \mathbb{C}^{N_{T} \times r}$ and $\mathbf{U} \in \mathbb{C}^{N_{R} \times r}$ are unitary matrices and $\boldsymbol{\Lambda} \in \mathbb{C}^{r \times r}$ is the diagonal matrix consisting of the $r$ nonzero eigenvalues of $\mathbf{H}$ in decreasing order of magnitude. Ignoring the rate constraint $(\mathrm{C} 1)$ in $\mathcal{O P} 1$ (or equivalently in $\mathcal{O P}$ ) leads to the rank-1 optimal TX covariance matrix $\mathbf{S}^{*}=\mathbf{S}_{\mathrm{EB}} \triangleq P_{T} \mathbf{v}_{1} \mathbf{v}_{1}^{\mathrm{H}}$ [12], [30], where $\mathbf{v}_{1} \in \mathbb{C}^{N_{T} \times 1}$ is the first column of $\mathbf{V}$ that corresponds to the eigenvalue $[\boldsymbol{\Lambda}]_{1,1} \triangleq$ $\sqrt{\lambda_{\max }\left(\mathbf{H}^{\mathrm{H}} \mathbf{H}\right)}$. This TX precoding, also known as transmit energy beamforming, allocates $P_{T}$ to the strongest eigenmode of $\mathbf{H}^{\mathrm{H}} \mathbf{H}$ and is known to maximize the harvested or received power. On the other hand, it is also well known [31] that one may profit from the existence of multiple antennas and channel estimation techniques to realize spatial multiplexing of multiple data streams. Spatial multiplexing adopts the

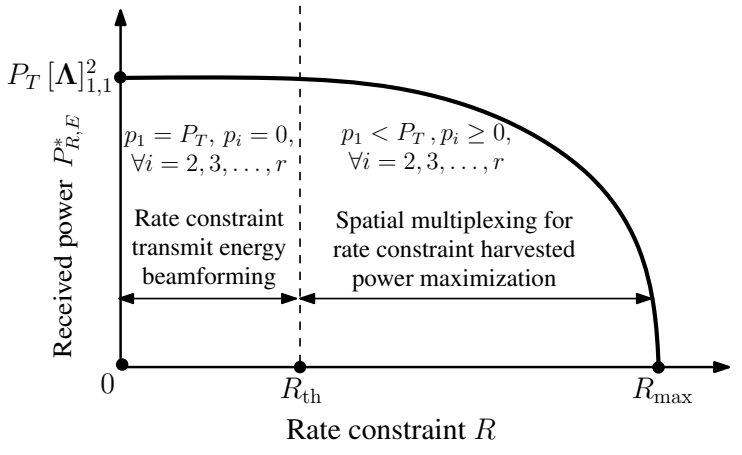

Fig. 3. Trade off between received power for EH and achievable information rate. $R_{\mathrm{th}}$ is the switching point between TX precoding modes: energy beamforming and information spatial multiplexing.

waterfilling technique to perform optimal allocation of $P_{T}$ over all the available eigenchannels of MIMO channel matrix. Evidently, for our problem formulation $\mathcal{O P} 1$ including the rate constraint $(\mathrm{C} 1)$ and PS reception, we need to investigate the underlying fundamental trade off between TX energy beamforming and information spatial multiplexing. As previously described, these two transmission schemes have contradictory objectives, and thus provide different TX designs.

Suppose we adopt energy beamforming in $\mathcal{O P} 1$, resulting in the received RF power $P_{R_{\mathrm{EB}}} \triangleq \rho_{\mathrm{EB}} P_{T}[\boldsymbol{\Lambda}]_{1,1}^{2}$ where $\rho_{\mathrm{EB}}$ represents the unknown UPS parameter. To find the optimal UPS parameter $\rho_{\mathrm{EB}}^{*}$, we need to seek for the best power allocation $\left(1-\rho_{\mathrm{EB}}^{*}\right)$ for ID meeting the rate requirement $R$. To do so, we solve (C1) at equality over UPS parameter yielding

$$
\rho_{\mathrm{EB}}^{*} \triangleq \max \left\{0,1-\left(2^{R}-1\right) \sigma^{2}\left[P_{T}[\boldsymbol{\Lambda}]_{1,1}^{2}\right]^{-1}\right\} .
$$

It can be concluded that both $\rho_{\mathrm{EB}}^{*}$ and the maximum received $\mathrm{RF}$ power given by $\rho_{\mathrm{EB}}^{*} P_{T}[\boldsymbol{\Lambda}]_{1,1}^{2}$ are decreasing functions of $R$. This reveals that there exists a rate threshold $R_{\mathrm{th}}$ such that, when $R>R_{\mathrm{th}}$, one should allocate $P_{T}$ over to at least two eigenchannels instead of performing energy beamforming, i.e., instead of assigning $P_{T}$ solely to the strongest eigenchannel. We are henceforth interested in finding this $R_{\text {th }}$ value. Consider the optimum power allocation $p_{1}^{*}$ and $p_{2}^{*}$ for the two highest gained eigenchannels with eigenmodes $[\boldsymbol{\Lambda}]_{1,1}$ and $[\boldsymbol{\Lambda}]_{2,2}$, respectively, with $[\boldsymbol{\Lambda}]_{1,1}>$ $[\boldsymbol{\Lambda}]_{2,2}$. By substituting these values into $(\mathrm{C} 1)$, defining $\mathcal{Z}_{1} \triangleq$ $\sqrt{\left([\boldsymbol{\Lambda}]_{1,1}^{2} p_{1}^{*}-[\boldsymbol{\Lambda}]_{2,2}^{2} p_{2}^{*}\right)^{2}+2^{R+2}[\boldsymbol{\Lambda}]_{1,1}^{2}[\boldsymbol{\Lambda}]_{2,2}^{2} p_{1}^{*} p_{2}^{*}}$, and solving at equality for the optimum UPS parameter $\rho_{\mathrm{SM}_{2}}^{*}$ for spatial multiplexing over two eigenchannels deduces to

$$
\rho_{\mathrm{SM}_{2}}^{*} \triangleq 1+\frac{\sigma^{2}}{2}\left(\frac{1}{[\boldsymbol{\Lambda}]_{1,1}^{2} p_{1}^{*}}+\frac{1}{[\mathbf{\Lambda}]_{2,2}^{2} p_{2}^{*}}+\frac{\mathcal{Z}_{1}}{[\boldsymbol{\Lambda}]_{1,1}^{2}[\boldsymbol{\Lambda}]_{2,2}^{2} p_{1}^{*} p_{2}^{*}}\right)
$$

resulting in the maximum received $\mathrm{RF}$ power for $\mathrm{EH}$ given by $\rho_{\mathrm{SM}_{2}}^{*}\left(p_{1}^{*}[\boldsymbol{\Lambda}]_{1,1}^{2}+p_{2}^{*}[\boldsymbol{\Lambda}]_{2,2}^{2}\right)$. We now combine the latterly obtained maximum received RF power with spatial multiplexing and that of energy beamforming to compute $R_{\mathrm{th}}$. The rate threshold value that renders energy beamforming more 
beneficial than spatial multiplexing in terms of received RF power can be obtained from solution of following inequality

$$
\rho_{\mathrm{EB}}^{*} P_{T}[\boldsymbol{\Lambda}]_{1,1}^{2}>\rho_{\mathrm{SM}_{2}}^{*}\left(p_{1}^{*}[\boldsymbol{\Lambda}]_{1,1}^{2}+p_{2}^{*}[\boldsymbol{\Lambda}]_{2,2}^{2}\right) .
$$

Substituting (3) and (4) into (5), defining $\mathcal{Z}_{2} \triangleq\left([\boldsymbol{\Lambda}]_{1,1}^{2}-\right.$ $\left.[\boldsymbol{\Lambda}]_{2,2}^{2}\right)\left([\boldsymbol{\Lambda}]_{1,1}^{2} p_{1}^{*}+p_{2}^{*}[\boldsymbol{\Lambda}]_{2,2}^{2}\right)^{2}$, and applying some algebraic manipulations yields the desired threshold in closed-form:

$R_{\mathrm{th}} \triangleq \log _{2}\left(1+\frac{p_{2}^{*}\left([\boldsymbol{\Lambda}]_{1,1}^{2}-[\boldsymbol{\Lambda}]_{2,2}^{2}\right)}{\sigma^{2}}+\sqrt{\frac{\mathcal{Z}_{2}}{[\boldsymbol{\Lambda}]_{1,1}^{2}[\boldsymbol{\Lambda}]_{2,2}^{2} \sigma^{2} p_{1}^{*}}}\right)$

Remark 1: The rate threshold $R_{\text {th }}$ given by (6) evinces a switching point on the desired TX precoding operation, which is graphically presented in Fig. 3. When the rate requirement $R$ is less or equal to $R_{\mathrm{th}}$, energy beamforming is sufficient to meet $R$, and hence, can be used for maximizing the received $\mathrm{RF}$ power. For cases where $R>R_{\mathrm{th}}$, statistical multiplexing needs to be adopted for maximizing the received RF power for $\mathrm{EH}$ while satisfying $R$. This explicit non-trivial switching point $R_{\mathrm{th}}$ for the TX precoding mode is unique to the problem formulation considered in this paper, and has not been explored or investigated in the relevant literature [12], [16][22] for the complementary problem formulations therein.

We next use $R_{\mathrm{th}}$ in (6) to obtain solutions for $\mathcal{O P} 1$ and $\mathcal{O P} 2$.

\section{B. Globally Optimal Solution of $\mathcal{O P} 1$}

Associating Lagrange multipliers $\mu$ and $\nu$ with constraints (C1) and (C2), respectively, while keeping (C3) and (C4) implicit, the Lagrangian function of $\mathcal{O P} 1$ is defined as

$$
\mathcal{L}=\rho P_{R}-\nu\left(\operatorname{tr}(\mathbf{S})-P_{T}\right)-\mu\left(R-\log _{2}\left[\operatorname{det}\left(\mathcal{Z}_{3}\right)\right]\right) .
$$

where $\mathcal{Z}_{3} \triangleq\left(\mathbf{I}_{N_{R}}+(1-\rho) \sigma^{-2} \mathbf{H S H}^{\mathrm{H}}\right)$. Using Theorem 1, the global optimal solution $\left(\mathbf{S}^{*}, \rho^{*}\right)$ for $\mathcal{O P} 1$ is obtained from the following four Karush-Kuhn-Tucker (KKT) conditions (the subgradient and complimentary slackness conditions are defined, whereas the primal feasibility $(\mathrm{C} 1)-(\mathrm{C} 4)$ and dual feasibility constraints $\mu, \nu \geq 0$ are kept implicit):

$$
\begin{gathered}
\frac{\partial \mathcal{L}}{\partial \mathbf{S}}=\frac{\mu(1-\rho)}{\sigma^{2} \ln 2} \mathbf{H}^{\mathrm{H}} \mathcal{Z}_{3}^{-1} \mathbf{H}+\rho \mathbf{H}^{\mathrm{H}} \mathbf{H}-\nu \mathbf{I}_{N_{T}}=0 \\
\frac{\partial \mathcal{L}}{\partial \rho}=-\mu \operatorname{tr}\left(\frac{\mathbf{H} \mathbf{S H}^{\mathrm{H}}}{\sigma^{2} \ln 2} \mathcal{Z}_{3}^{-1}\right)+\operatorname{tr}\left(\mathbf{H S} \mathbf{H}^{\mathrm{H}}\right)=0 \\
\mu\left(R-\log _{2}\left[\operatorname{det} \mathcal{Z}_{3}\right]\right)=0 \\
\nu\left(\operatorname{tr}(\mathbf{S})-P_{T}\right)=0 .
\end{gathered}
$$

Solving (8) yields the KKT point [27], [29] defined by the optimal solution $\left(\mathbf{S}^{*}, \rho^{*}, \mu^{*}, \nu^{*}\right)$. It is noted that it must hold $\nu \neq 0$, because the total available transmit power $P_{T}$ is always fully utilized due to the monotonically increasing nature of the objective function $P_{R, E}$ in $\mathbf{S}$. This implies that $\operatorname{tr}(\mathbf{S})=$ $P_{T}$, which means that constraint $(\mathrm{C} 2)$ is always satisfied at equality. Similarly, it must hold $\mu \neq 0$, because the received RF power is strictly increasing in $\rho$ and, as such, the fraction $1-\rho$ allocated for ID needs to be sufficient in meeting (C1).

Recalling the trade off discussion in Section IV-A, when $R \leq R_{\mathrm{th}}$, the optimal TX covariance matrix is given as $\mathbf{S}^{*}=$
$\mathrm{S}_{\mathrm{EB}}$. For this case the optimum TX precoding operation is energy beamforming, i.e., $\mathbf{F} \triangleq \mathbf{V}\left(\mathbf{P}^{*}\right)^{1 / 2} \in \mathbb{C}^{N_{T} \times r}$ where the $r \times r$ matrix $\mathbf{P}^{*}$ is defined as $\mathbf{P}^{*}=\operatorname{diag}\left\{\left[P_{T} 0 \cdots 0\right]\right\}$, and the optimal UPS ratio is $\rho_{\mathrm{EB}}$ given by (3). Substituting $\mathbf{S}_{\mathrm{EB}}$ and $\rho_{\mathrm{EB}}$ into (8a) and (8b), yields corresponding multipliers:

$$
\begin{gathered}
\mu_{\mathrm{EB}} \triangleq \sigma^{2} \ln 2\left(1+\frac{\left(1-\rho_{\mathrm{EB}}\right) P_{T}[\boldsymbol{\Lambda}]_{1,1}^{2}}{\sigma^{2}}\right), \\
\nu_{\mathrm{EB}} \triangleq \frac{\mu_{\mathrm{EB}}\left(1-\rho_{\mathrm{EB}}\right)[\boldsymbol{\Lambda}]_{1,1}^{2}}{\ln (2)\left(\sigma^{2}+\left(1-\rho_{\mathrm{EB}}\right) P_{T}[\boldsymbol{\Lambda}]_{1,1}^{2}\right)}+[\boldsymbol{\Lambda}]_{1,1}^{2} \rho_{\mathrm{EB}} .
\end{gathered}
$$

We therefore conclude that $\left(\mathbf{S}^{*}, \rho^{*}, \mu^{*}, \nu^{*}\right)$ is given as $\left(\mathbf{S}_{\mathrm{EB}}, \rho_{\mathrm{EB}}, \mu_{\mathrm{EB}}, \nu_{\mathrm{EB}}\right)$ for $R \leq R_{\mathrm{th}}$. When $R>R_{\mathrm{th}}$, the optimum TX precoding operation is spatial multiplexing and we thus to obtain the TX covariance matrix, rewrite (8a) in the following form after applying algebraic simplifications.

$$
\boldsymbol{\Lambda} \mathbf{V}^{\mathrm{H}} \mathbf{S} \mathbf{V} \boldsymbol{\Lambda}=\frac{\mu}{\ln 2}\left(\nu \mathbf{I}_{r}-\rho \boldsymbol{\Lambda}^{\mathrm{H}} \boldsymbol{\Lambda}\right)^{-1} \boldsymbol{\Lambda}^{2}-\frac{\sigma^{2}}{1-\rho} \mathbf{I}_{r} .
$$

By performing the necessary left and right multiplications of (10) with $\Lambda^{-1}, \mathbf{V}$, and $\mathbf{V}^{\mathrm{H}}$ and setting $\rho, \mu$, and $\nu$ to their optimal values $\rho_{\mathrm{SM}}, \mu_{\mathrm{SM}}$, and $\nu_{\mathrm{SM}}$ for spatial multiplexing, the optimal TX covariance matrix for $R>R_{\mathrm{th}}$ can be derived as:

$$
\mathbf{S}_{\mathrm{SM}}=\mathbf{V}\left(\frac{\mu_{\mathrm{SM}}\left(\nu_{\mathrm{SM}} \mathbf{I}_{r}-\rho_{\mathrm{SM}} \boldsymbol{\Lambda}^{\mathrm{H}} \boldsymbol{\Lambda}\right)^{-1}}{\ln 2}-\frac{\sigma^{2} \boldsymbol{\Lambda}^{-2}}{1-\rho_{\mathrm{SM}}}\right) \mathbf{V}^{\mathrm{H}}
$$

On simplifying (8b) to solve for the optimal $\mu_{\mathrm{SM}}$ yields

$$
\mu_{\mathrm{SM}}=\frac{\operatorname{tr}\left(\mathbf{H} \mathbf{S}_{\mathrm{SM}} \mathbf{H}^{\mathrm{H}}\right)}{\operatorname{tr}\left(\frac{\mathbf{H S}_{\mathrm{SM}} \mathbf{H}^{\mathrm{H}}}{\sigma^{2} \ln 2}\left(\mathbf{I}_{N_{R}}+\left(1-\rho_{\mathrm{SM}}\right) \sigma^{-2} \mathbf{H} \mathbf{S}_{\mathrm{SM}} \mathbf{H}^{\mathrm{H}}\right)^{-1}\right)} \text {. }
$$

Evidently from (11), $\mathbf{S}_{\mathrm{SM}} \in \mathbb{C}^{N_{T} \times N_{T}}$ can be expressed as $\mathbf{S}_{\mathrm{SM}} \triangleq \mathbf{V} \mathbf{P}^{*} \mathbf{V}^{\mathrm{H}}$ with the $r \times r$ matrix $\mathbf{P}^{*} \triangleq$ $\operatorname{diag}\left\{\left[p_{1}^{*} p_{2}^{*} \cdots p_{r}^{*}\right]\right\}$ representing the optimal power allocation matrix among H's eigenchannels, whose entries are given by

$p_{k}^{*}=\left(\frac{\mu^{*}}{\ln 2\left(\nu_{\mathrm{SM}}-\rho_{\mathrm{SM}}[\boldsymbol{\Lambda}]_{k, k}^{2}\right)}-\frac{\sigma^{2}}{\left(1-\rho_{\mathrm{SM}}\right)[\boldsymbol{\Lambda}]_{k, k}^{2}}\right)^{+}$.

The optimal $\rho_{\mathrm{SM}}, \mu_{\mathrm{SM}}$, and $\nu_{\mathrm{SM}}$ is the solution of the system with the three equations (8c), (8d), and (12) after setting $\mathbf{S}=\mathbf{S}_{\mathrm{SM}}$ and satisfying $\mu, \nu>0$ and $0 \leq \rho<1$. Later in Section VI we first reduce this system of equations to two, and then by exploiting the tight bounds on $\nu^{*}$ derived in Section V-A2, we present how it can be efficiently implemented.

Remark 2: Observing (11) and (13) leads to the conclusion that the optimum TX precoding for $R>R_{\text {th }}$ is $\mathbf{F}=$ $\mathbf{V}\left(\mathbf{P}^{*}\right)^{1 / 2}$ with the $r$ diagonal elements of $\mathbf{P}^{*}$ given by (13). This precoding results in $r$ parallel eigenchannel transmissions with power allocation obtained from a modified waterfilling algorithm, where water levels depend on $R, P_{T}, \mathbf{H}$, and $\sigma^{2}$.

By combining these results for energy beamforming and spatial multiplexing, the optimal solution of $\mathcal{O P} 1$ is given by

$$
\mathbf{S}^{*}= \begin{cases}P_{T} \mathbf{v}_{1} \mathbf{v}_{1}^{\mathrm{H}}, & R \leq R_{\mathrm{th}} \leq R_{\max }, \\ \mathbf{V}\left(\frac{\mu^{*} \mathcal{Z}_{4}}{\ln 2}-\frac{\sigma^{2} \boldsymbol{\Lambda}^{-2}}{1-\rho}\right) \mathbf{V}^{\mathrm{H}}, & R_{\mathrm{th}}<R \leq R_{\max }, \\ \text { Infeasible, } & R>R_{\max },\end{cases}
$$


where $\mathcal{Z}_{4} \triangleq\left(\nu^{*} \mathbf{I}_{r}-\rho \boldsymbol{\Lambda}^{\mathrm{H}} \boldsymbol{\Lambda}\right)^{-1}, \rho^{*}=\rho_{\mathrm{EB}}, \mu^{*}=\mu_{\mathrm{EB}}$, and $\nu^{*}=\nu_{\mathrm{EB}}$ for $R \leq R_{\mathrm{th}}$, and for $R>R_{\mathrm{th}}, \rho^{*}=\rho_{\mathrm{SM}}, \mu^{*}=$ $\mu_{\mathrm{SM}}$, and $\nu^{*}=\nu_{\mathrm{SM}}$ are obtained from the solution of the system of equations described below (13). The feasibility of $\mathcal{O P} 1$ depends on $R_{\text {max }} \triangleq \log _{2}\left(\operatorname{det}\left(\mathbf{I}_{N_{R}}+\sigma^{-2} \mathbf{H S}_{\mathrm{wF}} \mathbf{H}^{\mathrm{H}}\right)\right)$, which represents the maximum achievable rate for UPS ratio $\rho=0$ and $\mathbf{S}_{\mathrm{WF}} \triangleq \mathbf{V} \mathbf{P}_{\mathrm{WF}} \mathbf{V}^{\mathrm{H}}$. In the latter expression, $\mathbf{P}_{\mathrm{WF}} \triangleq$ $\operatorname{diag}\left\{\left[p_{\mathrm{WF}, 1} p_{\mathrm{WF}, 2} \cdots p_{\mathrm{WF}, r}\right]\right\}$ is the $r \times r$ power allocation matrix whose rank $r_{w}$ (non-zero diagonal entries) is [32]

$$
r_{w} \triangleq \max \left\{k \mid P_{T}-\sum_{i=1}^{k-1}\left(\frac{\sigma^{2}}{[\boldsymbol{\Lambda}]_{k, k}^{2}}-\frac{\sigma^{2}}{[\boldsymbol{\Lambda}]_{i, i}^{2}}\right)>0, k \leq r\right\}
$$

and its non-zero elements with index $k=1,2, \ldots, r_{w}$ are obtained from the standard waterfilling algorithm as

$p_{\mathrm{WF}, k}=\frac{P_{T}-\sum_{i=1}^{r_{w}-1}\left(\frac{\sigma^{2}}{[\boldsymbol{\Lambda}]_{r_{w}, r_{w}}^{2}}-\frac{\sigma^{2}}{[\boldsymbol{\Lambda}]_{i, i}^{2}}\right)}{r_{w}}+\frac{\sigma^{2}}{[\boldsymbol{\Lambda}]_{r_{w}, r_{w}}^{2}}-\frac{\sigma^{2}}{[\boldsymbol{\Lambda}]_{k, k}^{2}}$.

Here we would like to add that based on (14) deciding whether the optimal TX precoding matrix $\mathbf{S}^{*}$ is denoted $\mathbf{S}_{\mathrm{EB}}$ or $\mathbf{S}_{\mathrm{SM}}$, the corresponding optimal TX signal vector $\mathbf{x}^{*} \in \mathbb{C}^{N_{T} \times 1}$ can be obtained as $\mathbf{x}_{\mathrm{EB}} \triangleq \sqrt{P_{T}} \mathbf{v}_{1} \widetilde{x}$ and $\mathbf{x}_{\mathrm{SM}} \triangleq \mathbf{V}\left(\mathbf{P}^{*}\right)^{1 / 2} \widetilde{\mathbf{x}}$. Here $\widetilde{x}$ is an arbitrary ZMCSCG signal and $\widetilde{\mathbf{x}} \in \mathbb{C}^{r \times 1}$ is a ZMCSCG random vector, both having unit variance entries.

\section{Globally Optimal Solution of $\mathcal{O P} 2$}

Like $\mathcal{O P} 1$, the proposed rate threshold value in $\mathcal{O P} 2$ determining the optimal TX precoding operation, is given by

$$
R_{\mathrm{th}}^{\mathrm{id}} \triangleq \log _{2}\left(1+\sigma^{-2} P_{T}[\boldsymbol{\Lambda}]_{1,1}^{2}\right)
$$

represents rate achieved by energy beamforming for ideal RX.

Lemma 2: Global optimal solution $\mathbf{S}_{\mathrm{id}}^{*}$ of $\mathcal{O P} 2$ is given by

$$
\mathbf{S}_{\mathrm{id}}^{*}= \begin{cases}P_{T} \mathbf{v}_{1} \mathbf{v}_{1}^{\mathrm{H}}, & R \leq R_{\mathrm{th}}^{\mathrm{id}} \leq R_{\max }, \\ \mathbf{V} \operatorname{diag}\left\{\left[p_{1}^{(\mathrm{id})} p_{2}^{(\mathrm{id})} \cdots p_{r}^{(\mathrm{id})}\right]\right\} \mathbf{V}^{\mathrm{H}}, & R_{\mathrm{th}}^{\mathrm{id}}<R \leq R_{\max }, \\ \text { Infeasible }, & R>R_{\max },\end{cases}
$$

with $p_{k}^{(\mathrm{id})}$ denoting power assignment to $k$-th eigenchannel is

$$
p_{k}^{(\mathrm{id})}=\left(\frac{\mu_{2}^{*}}{\ln 2\left(\nu_{2}^{*}-[\boldsymbol{\Lambda}]_{k, k}^{2}\right)}-\frac{\sigma^{2}}{[\boldsymbol{\Lambda}]_{k, k}^{2}}\right)^{+}, \forall k=1,2, \ldots, r .
$$

In the latter expression, $\nu_{2}^{*} \geq 0$ and $\mu_{2}^{*} \geq 0$ represent the Lagrange multipliers corresponding to constraints $(\mathrm{C} 2)$ and (C5), respectively. These can be obtained using a subgradient method as described in [12, App. A] such that $\log _{2}\left(\operatorname{det}\left(\mathbf{I}_{N_{R}}+\sigma^{-2} \mathbf{H S}_{\mathrm{id}}^{*} \mathbf{H}^{\mathrm{H}}\right)\right)=R$ and $\operatorname{tr}\left(\mathbf{S}_{\mathrm{id}}^{*}\right)=P_{T}$.

Proof: The proof is provided in Appendix A.

Remark 3: It can be observed from the solution of $\mathcal{O P} 2$ that our proposed TX precoding design significantly differs from that obtained from the solution of optimization problem (P3) in [12]. This reveals that the design maximizing the total received RF power for $\mathrm{EH}$, while satisfying a minimum instantaneous rate requirement, is very different from the design that maximizes the instantaneous rate subject to a minimum constraint on the total received RF power.

\section{Analytical Bounds And Asymptotic Relaxation}

Here we first present analytical bounds for the UPS ratio $\rho$ and Lagrange multipliers $\mu, \nu$ defined in Section IV. Then tight asymptotic approximations for the joint design are presented.

\section{A. Analytical Bounds}

1) UPS Ratio $\rho$ : The information rate is given by $\log _{2}\left(\operatorname{det}\left(\mathbf{I}_{N_{R}}+(1-\rho) \sigma^{-2} \mathbf{H S H}^{\mathrm{H}}\right)\right)$, which is a monotonically decreasing function of $\rho$. The upper bound $\rho_{\mathrm{UB}}$ on the feasible $\rho$ value satisfying $(\mathrm{C} 1)$ is given by the UPS ratio corresponding to the maximum achievable rate value $R_{\max }$ as achieved with statistical multiplexing over all available eigenchannels. Mathematically, $\rho_{\mathrm{UB}}$ as obtained by setting $\mathbf{S}=\mathbf{V} \mathbf{P}_{\mathrm{WF}} \mathbf{V}^{\mathrm{H}}$ with the entries of $\mathbf{P}_{\mathrm{WF}}$ defined in (16), is

$\rho_{\mathrm{UB}} \triangleq\left\{\rho \mid \operatorname{det}\left(\mathbf{I}_{N_{R}}+\frac{(1-\rho) \mathbf{H S}_{\mathrm{WF}} \mathbf{H}^{\mathrm{H}}}{\sigma^{2}}\right)=2^{R}, \rho \leq 1\right\}$.

Likewise, the lower bound on the feasible $\rho$ meeting (C1) is given by the UPS ratio $\rho_{\mathrm{EB}}$ defined in (3). This lower bound happens with energy beamforming, where entire TX power is allocated to the best gain eigenchannel and the achievable rate is minimum. Combining these results, yields $\rho_{\mathrm{EB}} \leq \rho \leq \rho_{\mathrm{UB}}$.

2) Lagrange Multipliers $\mu$ and $\nu$ for $R>R_{\mathrm{th}}$ : To have non-negative power allocation $p_{1}$ over the best gain eigenchannel having eigenmode $[\boldsymbol{\Lambda}]_{1,1}$, it must hold from (13) that $\nu \geq \nu_{\mathrm{LB}} \triangleq \rho[\boldsymbol{\Lambda}]_{1,1}^{2}$. Also, using the definition $p_{1}=\alpha P_{T}$ with $\alpha \leq 1$ in (13) for $k=1$ yields $\mu=$ $\left(\nu-\rho[\boldsymbol{\Lambda}]_{1,1}^{2}\right)\left(\alpha P_{T}+\frac{\sigma^{2}}{(1-\rho)[\boldsymbol{\Lambda}]_{1,1}^{2}}\right) \ln 2$. Since for the total received power holds $\operatorname{tr}\left(\mathbf{H S H}^{\mathrm{H}}\right) \leq P_{T}$ and also (12) holds, the upper bound for $\mu$, denoted by $\mu_{\mathrm{UB}}$, can be obtained as

$$
\mu<\frac{(a)}{(1-\rho) \ln 2 \operatorname{tr}\left(\mathbf{H S H}^{\mathrm{H}}\right)} \underset{r}{r}<\mu_{\mathrm{UB}} \triangleq \frac{(1-\rho) P_{T} \ln 2}{r}
$$

where $(a)$ results from the high SNR approximation. Combining (21) with $\frac{\sigma^{2}}{(1-\rho)[\boldsymbol{\Lambda}]_{1,1}^{2}}>0$, leads to $\nu<\frac{(1-\rho)}{\alpha r}+\rho[\boldsymbol{\Lambda}]_{1,1}^{2}$. Due to the highest power allocation over the best gain eigenchannel, it must hold $\alpha \geq \frac{1}{r}$, yielding $\nu_{\mathrm{UB}} \triangleq 1+\rho\left([\boldsymbol{\Lambda}]_{1,1}^{2}-1\right)$. However as shown later, $\nu \ll \nu_{\mathrm{UB}}$ because the total received power $P_{R} \ll P_{T}$. These bounds will be used in Section VI-B for efficiently implementing the global optimization algorithm.

\section{B. Asymptotic Analysis}

The received RF power for EH in SWIPT systems needs to be greater than energy reception sensitivity [2], [3], which is in the order of $-10 \mathrm{dBm}$ to $-30 \mathrm{dBm}$, for having nonzero harvested DC power after rectification. Since, the AWGN power spectral density is around $-175 \mathrm{dBm} / \mathrm{Hz}$ leading to an average received noise power of around $-100 \mathrm{dBm}$ for SWIPT at $915 \mathrm{MHz}$, the received SNR in practical SWIPT systems is very high, i.e., around $70 \mathrm{~dB}$, even for very high frequency transmissions. Based on this practical observation for SWIPT, we next investigate the joint design for high SNR scenarios. 
1) Globally Optimal Solution of $\mathcal{O P} 1$ for High SNR: The optimal solution of $\mathcal{O P} 1$ for high SNR values defined as $\left(\mathbf{S}_{\mathbf{a}}^{*}, \rho_{a}^{*}, \mu_{a}^{*}, \nu_{a}^{*}\right)$ can be obtained similarly to Section IV-B as

$\mathbf{S}_{\mathrm{a}}^{*}= \begin{cases}P_{T} \mathbf{v}_{1} \mathbf{v}_{1}^{\mathrm{H}}, & R \leq R_{\mathrm{th}} \leq R_{\max }, \\ \mathbf{V}\left(\frac{\mu_{a}^{*}\left(\nu_{a}^{*} \mathbf{I}_{r}-\rho_{a}^{*} \boldsymbol{\Lambda}^{\mathrm{H}} \boldsymbol{\Lambda}\right)^{-1}}{\ln 2}\right) \mathbf{V}^{\mathrm{H}}, & R_{\mathrm{th}}<R \leq R_{\max }, \\ \text { Infeasible, } & R>R_{\max },\end{cases}$

$\mu_{a}^{*}=r^{-1}\left(1-\rho_{a}^{*}\right) \operatorname{tr}\left(\mathbf{H S}_{\mathbf{a}}^{*} \mathbf{H}^{\mathrm{H}}\right) \ln 2$, and the remaining two unknowns $\rho_{a}^{*}$ and $\nu_{a}^{*}$ are given from the solutions of the equations $\operatorname{tr}\left(\mathbf{S}_{\mathbf{a}}^{*}\right)=P_{T}$ and $\log _{2}\left(\operatorname{det}\left(\sigma^{-2}(1-\rho) \mathbf{H S}_{\mathbf{a}}^{*} \mathbf{H}^{\mathrm{H}}\right)\right)=$ $R$. After some simplifications with (22), the power allocation is obtained as $p_{a, k}^{*}=\frac{\mu_{a}^{*}}{\left(\nu_{a}^{*}-\rho_{a}^{*}[\boldsymbol{\Lambda}]_{k, k}^{2}\right) \ln 2}, \forall k=1,2, \ldots, r$. Hence, under high SNR, the optimal power allocation over available eigenchannels for $R>R_{\mathrm{th}}$ is always greater than zero regardless of the relative strengths of the eigenmodes.

2) Globally Optimal Solution of $\mathcal{O P} 2$ for High SNR: Using the derived analytical bounds for $\rho$ and $\nu$ along with Lemma 2, the asymptotic approximation $\mathbf{S}_{\mathrm{id}, \mathrm{a}}^{*}$ for $\mathbf{S}_{\mathrm{id}}^{*}$ can be obtained as $\mathbf{S}_{\mathrm{id}, \mathrm{a}}^{*}= \begin{cases}P_{T} \mathbf{v}_{1} \mathbf{v}_{1}^{\mathrm{H}}, & R \leq R_{\mathrm{th}}^{\mathrm{id}} \leq R_{\max }, \\ \mathbf{V} \operatorname{diag}\left\{\left[p_{1}^{(\mathrm{id}, \mathrm{a})} \cdots p_{r}^{(\mathrm{id}, \mathrm{a})}\right]\right\} \mathbf{V}^{\mathrm{H}}, & R_{\mathrm{th}}^{\mathrm{id}}<R \leq R_{\max }, \\ \text { Infeasible, } & R>R_{\max },\end{cases}$ where each $p_{k}^{(\mathrm{id}, \mathrm{a})}$ with $k=1,2, \ldots, r$ is given by

$$
p_{k}^{(\mathrm{id}, \mathrm{a})}=\frac{\mu_{2 a}^{*}}{\left(\nu_{2 a}^{*}-[\mathbf{\Lambda}]_{k, k}^{2}\right) \ln 2} .
$$

With $[\boldsymbol{\Lambda}]_{1,1}^{2}<\nu_{2 a}^{*}<[\boldsymbol{\Lambda}]_{1,1}^{2}+1$, solving $\sum_{k=1}^{r} p_{k}^{(\mathrm{id}, \mathrm{a})}=P_{T}$ yields $\mu_{2 a}^{*}=P_{T}\left(\sum_{k=1}^{r} \frac{1}{\left(\nu_{2 a}^{*}-[\boldsymbol{\Lambda}]_{k, k}^{2}\right) \ln 2}\right)^{-1}$. Setting $\beta \triangleq \nu_{2 a}^{*}-$ $[\boldsymbol{\Lambda}]_{1,1}^{2}$ and substituting in (24), we can rewrite $p_{k}^{(\mathrm{id}, \mathrm{a})}$ as

$$
p_{k}^{(\mathrm{id}, \mathrm{a})}=\beta p_{1}^{\text {(id,a) }}\left[\beta+[\boldsymbol{\Lambda}]_{1,1}^{2}-[\mathbf{\Lambda}]_{k, k}^{2}\right]^{-1} .
$$

To solve for $\beta \in(0,1)$, we need to replace into the rate constraint expression leading to $\prod_{k=1}^{r}\left(\frac{p_{k}^{(\mathrm{id}, \mathrm{a})}[\boldsymbol{\Lambda}]_{k, k}^{2}}{\sigma^{2}}\right)=2^{R}$, which after some simplifications results in the expression

$$
\prod_{k=1}^{r}\left(\frac{\beta[\boldsymbol{\Lambda}]_{k, k}^{2}}{\beta+[\boldsymbol{\Lambda}]_{1,1}^{2}-[\boldsymbol{\Lambda}]_{k, k}^{2}}\right)=2^{R}\left(\frac{\sigma^{2}}{p_{1}^{(\mathrm{id}, \mathrm{a})}}\right)^{r} .
$$

The $p_{1}^{(\mathrm{id}, \mathrm{a})}$ in (26) can be obtained in closed-form as a function of $\beta$ by solving $\sum_{k=1}^{r} p_{k}^{(\mathrm{id}, \mathrm{a})}=P_{T}$ and using (25), yielding

$$
p_{1}^{(\mathrm{id}, \mathrm{a})}=P_{T}\left(1+\sum_{k=2}^{r}\left(\frac{\beta}{\beta+[\boldsymbol{\Lambda}]_{1,1}^{2}-[\mathbf{\Lambda}]_{k, k}^{2}}\right)\right)^{-1} .
$$

Using these developments in Section VI-B3 we show that the asymptotically optimal TX precoding for $\mathcal{O P} 2$ can be obtained using a 1-D linear search over very short range $(0,1)$ of $\beta$.

Remark 4: With the expressions (26) and (27) resulted from our derived asymptotic analysis, we have managed to replace the problem of finding the positive real values of $\mu_{2}$ and $\nu_{2}$ in $\mathcal{O P} 2$ along with the required waterfilling-based decision making process (involving a discontinuous function $(x)^{+}$due to underlying (C3)) by a simple linear search over $\beta \in(0,1)$.

\section{EfFicient Global Optimization Algorithm}

The goal here is to first present a global optimization algorithm to obtain the previously derived optimal solutions for $\mathcal{O P} 1$ and $\mathcal{O P} 2$ by effectively solving the KKT conditions. After that we present an alternate low complexity algorithm based on a simple 2-D linear search to practically implement the former algorithm in a computational efficient manner.

\section{A. Solving the KKT Conditions}

As discussed in Section IV, $\mathbf{S}^{*}$ and $\rho^{*}$ of $\mathcal{O P} 1$ for $R>R_{\mathrm{th}}$ are obtained by solving (8c), (8d), and (12) for $\rho^{*}, \mu^{*}$, and $\nu^{*}$ after setting $\mathbf{S}=\mathbf{S}_{\mathrm{SM}}$. Likewise, from Lemma $2, \mathbf{S}_{\mathrm{id}}^{*}$ of $\mathcal{O P} 2$ for $R>R_{\mathrm{th}}^{\text {id }}$ is derived by solving $\log _{2}\left(\operatorname{det}\left(\mathbf{I}_{N_{R}}+\frac{\mathbf{H S}_{\mathrm{id}}^{*} \mathbf{H}^{\mathrm{H}}}{\sigma^{2}}\right)\right)=R$ and $\operatorname{tr}\left(\mathbf{S}_{\mathrm{id}}^{*}\right)=P_{T}$ in $\mu_{2}^{*}$ and $\nu_{2}^{*}$.

1) Reduction of the System of Non-linear Equations: It is in general very difficult to efficiently solve a large system of non-linear equations. Hereinafter, we discuss the reduction of the number of the non-linear equations to be solved from three to two in $\mathcal{O P} 1$ and from two to one in $\mathcal{O P} 2$.

Let us denote the rank of the optimal TX covariance matrix by $r_{s}$. It represents the number of eigenchannels that have non-zero power allocation, i.e., $p_{k}>0$ with $k=1,2, \ldots, r_{s}$. Substituting this definition into (8d) and (13) with $\nu>0$, we can express $\mu^{*}$ in terms of $\nu^{*}$ and $\rho^{*}$ as

$$
\mu^{*}=\frac{P_{T}+\sum_{k=1}^{r_{s}} \sigma^{2}\left(\left(1-\rho^{*}\right)[\boldsymbol{\Lambda}]_{k, k}^{2}\right)^{-1}}{r_{s} \sum_{k=1}^{r_{s}}\left(\left(\nu^{*}-\rho^{*}[\boldsymbol{\Lambda}]_{k, k}^{2}\right) \ln 2\right)^{-1}} .
$$

Using definition of $r_{s}$ in (8c) and (13) with $\mu>0$, we obtain

$$
\mu^{*}=2^{\frac{R}{r_{s}}} \sigma^{2}\left(\left(1-\rho^{*}\right)\left(\prod_{k=1}^{r_{s}} \frac{[\boldsymbol{\Lambda}]_{k, k}^{2}}{\nu^{*}-\rho^{*}[\boldsymbol{\Lambda}]_{k, k}^{2}}\right)^{\frac{1}{r_{s}}}\right)^{-1} \ln 2 .
$$

By combining (12), (28), and (29), the reduced system of two non-linear equations to be solved for $\rho^{*}$ and $\nu^{*}$ as included in KKT point $\left(\mathbf{S}^{*}, \rho^{*}, \mu^{*}, \nu^{*}\right)$ for $R>R_{\mathrm{th}}$ in $\mathcal{O P} 1$ is given by

$$
\frac{P_{T}\left(1-\rho^{*}\right) \sigma^{-2}+\sum_{k=1}^{r_{s}}[\boldsymbol{\Lambda}]_{k, k}^{-2}}{2^{\frac{R}{r_{s}}} r_{s} \sum_{k=1}^{r_{s}}\left(\nu^{*}-\rho^{*}[\boldsymbol{\Lambda}]_{k, k}^{2}\right)^{-1}}=\left(\prod_{k=1}^{r_{s}} \frac{[\boldsymbol{\Lambda}]_{k, k}^{2}}{\nu^{*}-\rho^{*}[\boldsymbol{\Lambda}]_{k, k}^{2}}\right)^{-\frac{1}{r_{s}}}
$$

$\sum_{k=1}^{r_{s}} \frac{p_{k}^{*}[\boldsymbol{\Lambda}]_{k, k}^{2}\left(1-\rho^{*}\right)^{-1}}{1+\left(1-\rho^{*}\right) p_{k}^{*}[\boldsymbol{\Lambda}]_{k, k}^{2}}=\frac{\sum_{k=1}^{r_{s}} p_{k}^{*}[\boldsymbol{\Lambda}]_{k, k}^{2}\left(\prod_{j=1}^{r_{s}} \frac{[\boldsymbol{\Lambda}]_{j, j}^{2}}{\nu^{*}-\rho^{*}[\boldsymbol{\Lambda}]_{j, j}^{2}}\right)^{\frac{1}{r_{s}}}}{2^{\frac{R}{r_{s}}} \sigma^{2} \ln 2}$

where $p_{k}^{*}=\frac{\sigma^{2}}{\left(1-\rho^{*}\right)}\left(\frac{2^{\frac{R}{r_{s}}}\left(\nu^{*}-\rho^{*}[\Lambda]_{k, k}^{2}\right)^{-1}}{\left(\prod_{j=1}^{r_{s}} \frac{[\Lambda]_{j, j}^{2}}{\nu^{*}-\rho^{*}[\Lambda]_{j, j}^{2}}\right)^{\frac{1}{r_{s}}}}-\frac{1}{[\Lambda]_{k, k}^{2}}\right)^{+}, \forall k=$ $1,2, \ldots, r_{s}$. In a similar manner, the single non-linear equation that needs to be solved for computing $\nu_{2}^{*}$ included in the KKT point $\left(\mathbf{S}_{\mathrm{id}}^{*}, \mu_{2}^{*}, \nu_{2}^{*}\right)$ for $R>R_{\mathrm{th}}^{\mathrm{id}}$ in $\mathcal{O P} 2$ is given by

$$
\left(\frac{P_{T}}{\sigma^{2}}+\sum_{j=1}^{r_{s}} \frac{1}{[\boldsymbol{\Lambda}]_{j, j}^{2}}\right)\left(\prod_{k=1}^{r_{s}} \frac{[\boldsymbol{\Lambda}]_{k, k}^{2}}{\nu_{2}^{*}-[\boldsymbol{\Lambda}]_{k, k}^{2}}\right)^{\frac{1}{r_{s}}}=\sum_{k=1}^{r_{s}} \frac{2^{\frac{R}{r_{s}}} r_{s}}{\nu_{2}^{*}-[\boldsymbol{\Lambda}]_{k, k}^{2}} \text {. }
$$




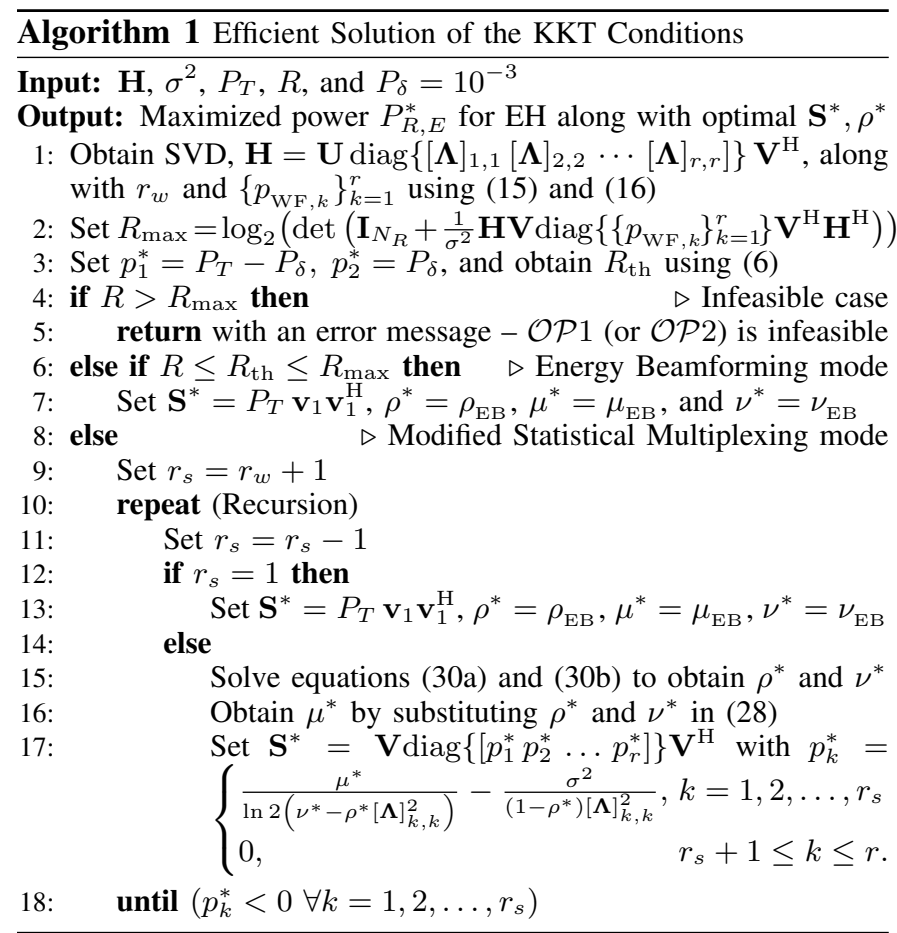

Lemma 3: The rank $r_{s}$ of the optimal TX covariance matrix $\mathbf{S}^{*}$ of $\mathcal{O P} 1$ (or $\mathbf{S}_{\mathrm{id}}^{*}$ of $\mathcal{O P} 2$ ) is always lower or equal to the rank $r_{w}$ of $\mathbf{S}_{\mathrm{WF}}$ providing the maximum achievable rate $R_{\max }$.

Proof: The proof follows from Section IV-B, where $P_{R, E}$ for $\mathrm{EH}$ is given by the rank-1 covariance matrix $\mathrm{S}_{\mathrm{EB}}$ implying TX energy beamforming. With increasing rate requirement $R>R_{\mathrm{th}}$, the optimal TX precoding switches from energy beamforming to statistical multiplexing. In this case, the power allocated over the best gain eigenchannel monotonically decreases due to the power allocation among the other available eigenchannels, thus increasing $r_{s}$ and decreasing $P_{R, E}$. The rate $R_{\max }$ is achieved by $\mathbf{S}_{\mathrm{wF}}$ having rank $r_{w} \leq r$, which also results in the minimum $P_{R, E}$ for both $\mathcal{O P} 1$ and $\mathcal{O P} 2$. Therefore, $r_{w}$ represents the maximum rank of the TX covariance matrix, hence it must hold $r_{s} \leq r_{w}$.

2) Implementation Details and Challenges: Here we first present the detailed steps involved in the implementation of solving the reduced system of non-linear equations to obtain the optimal design for both $\mathcal{O P} 1$ and $\mathcal{O P} 2$ via Algorithm 1. After that we discuss the practical challenges involved in implementing it directly using the commercial numerical solvers which may suffer from slow convergence issues as faced by the subgradient methods [12], [14], [16], [22] and semidefinite relaxations [17]-[21] used in the MIMO SWIPT literature.

From Algorithm 1, obtaining $\mathbf{S}^{*}$ and $\rho^{*}$ involves solving (30a) and (30b) for at most $r$ times, while considering positive power allocation over the $k$ best gain eigenchannels with $k=$ $1,2, \ldots, r$. Since (C3) and (C4) had been kept implicit, we repeatedly solve the latter system of equations for at most $r_{w} \leq r$ times till we obtain a feasible solution $\mathbf{S}^{*}$ and $\rho^{*}$.

Algorithm 1 can be slightly modified to provide the optimal solution of $\mathcal{O P} 2$. In particular, steps $7,13,15,16$, and 17 need to be updated for $\mathcal{O P} 2$. Starting with steps 7 and 13 , we need to remove $\rho^{*}$ since $\mathcal{O P} 2$ involves ideal reception and the optimal values of Lagrange multipliers $\mu_{2}$ and $\nu_{2}$ for $R \leq R_{\mathrm{th}}$ are given by $\mu_{2}^{*}=0$ and $\nu_{2}^{*}=[\boldsymbol{\Lambda}]_{1,1}^{2}$. In addition, to find $\nu_{2}^{*}$ for $R>R_{\mathrm{th}}$ in step 15 we need to solve (31). The solution $\nu_{2}^{*}$ of (31) needs then to update steps 16 and 17 in Algorithm 1, and the optimal $\mu_{2}^{*}$ and $p_{k}^{(\mathrm{id})}$,s can be derived as

$$
\begin{aligned}
& \mu_{2}^{*}=\left(P_{T}+\sum_{k=1}^{r_{s}} \frac{\sigma^{2}}{[\boldsymbol{\Lambda}]_{k, k}^{2}}\right)\left(r_{s} \sum_{k=1}^{r_{s}} \frac{1}{\left(\nu_{2}^{*}-[\boldsymbol{\Lambda}]_{k, k}^{2}\right) \ln 2}\right)^{-1} \\
& p_{k}^{(\mathrm{id})}= \begin{cases}\frac{\mu_{2}^{*}}{\left(\nu_{2}^{*}-[\boldsymbol{\Lambda}]_{k, k}^{2}\right) \ln 2}-\frac{\sigma^{2}}{[\boldsymbol{\Lambda}]_{k, k}^{2}}, & k=1,2, \ldots, r_{s} . \\
0, & r_{s}+1 \leq k \leq r\end{cases}
\end{aligned}
$$

The convergence of Algorithm 1 to its globally optimal solution is guaranteed due to its generalized convexity property [28], [29], as proved in Theorem 1. However, its speed of convergence depends on the efficiency of deployed numerical methods for solving (30a) and (30b). Commercial mathematical packages like Matlab provide very efficient solvers for such non-linear systems having unique solution. But convergence speed of those solvers or conventional subgradient methods [33] depends on the starting point and step sizes.

To characterize the exact number of computations required in achieving a desired level of accuracy with the derived globally optimal solutions, regardless of the starting point and step-sizes fed to the numerical solvers, we next present a simple, yet efficient, 2-D linear search algorithm based on the Golden Section Search (GSS) method [34] that provides an effective way of practically implementing Algorithm 1. We would like to mention that the main steps involved in the global optimization algorithm implemented using Algorithm 2 remain the same as in Algorithm 1. Except that it presents an efficient way of implementing step 15 of Algorithm 1.

\section{B. Two-Dimensional (2-D) Linear Search}

As discussed in Section III, for a known $\rho, \mathcal{O P} 1$ is a convex optimization problem having a linear objective and convex constraints. Using this property and the small feasible range of $\rho$ given by $0 \leq \rho_{\mathrm{LB}} \leq \rho \leq \rho_{\mathrm{UB}} \leq 1$ as derived in Section $\mathrm{V}$-A1, we propose to iteratively solve $\mathcal{O P} 1$ for a given $\rho$ value till the globally optimal $\left(\mathbf{S}^{*}, \rho^{*}\right)$ pair is obtained providing the unique maximum received power $P_{R, E}^{*}$. To traverse over the short value space of $\rho$ we use the GSS method [34] that provides fast convergence to the unique root of an equation or a globally optimal solution of a unimodal function. For each feasible $\rho$ value, we substitute into (30a) and then solve it for the optimal $\nu^{*}$. As shown in Section V-A2, $\nu_{\mathrm{UB}}-\nu_{\mathrm{LB}}=1-\rho \leq 1$ implying that the search space for the optimal $\nu^{*}$ is very small. Thus, (30a) can be solved very efficiently for $\nu^{*}$ for a given $\rho^{*}$ value by using the standard one-dimensional (1-D) GSS method or conventional root finding techniques available in commercial softwares.

1) Implementation Details: The algorithmic steps for the proposed 2-D GSS solution are summarized in Algorithm 2 which includes two linear searches. An outer search aiming at finding $\rho^{*}$ and an inner one to seek for $\nu^{*}$ for each given $\rho$ value. Due to the implicit consideration of (C3), obtaining $\nu^{*}$ for a given $\rho^{*}$ involves solving (30a) using 1-D GSS 


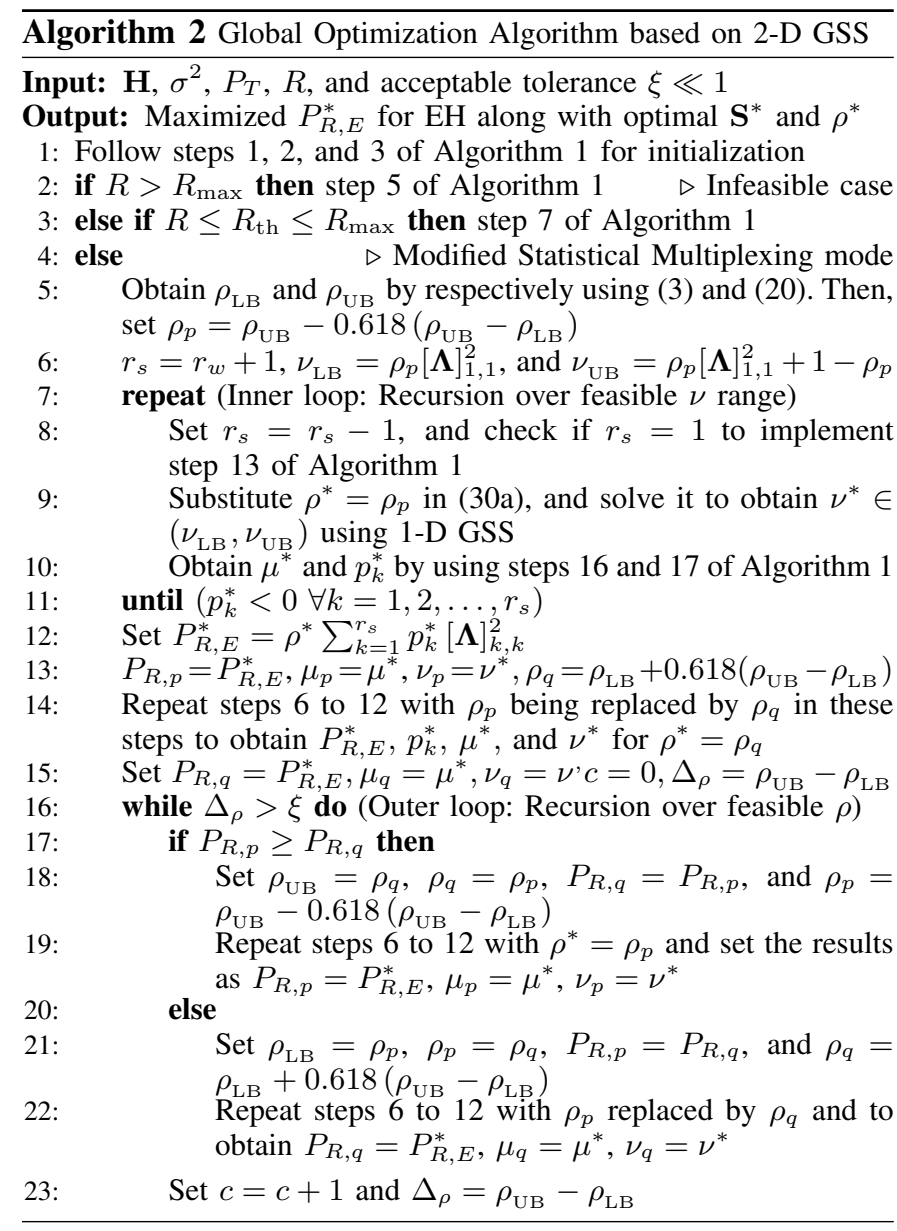

for at most $r_{w} \leq r$ times, while considering positive power allocation over the $k=1,2, \ldots, r$ best gain eigenchannels.

Algorithm 2 can also be slightly modified to be used for obtaining the solution for $\mathcal{O P} 2$, where due to ideal reception, the outer GSS over the feasible $\rho$ values has to be removed and we only need to perform a 1-D GSS for $\nu_{2}^{*}$ over its feasible value range $[\boldsymbol{\Lambda}]_{1,1}^{2} \leq \nu_{2}^{*} \leq[\boldsymbol{\Lambda}]_{1,1}^{2}+1$. So, for $\mathcal{O P} 2$ we need to consider steps 1-12 of Algorithm 2, excluding the initialization step 5, and updating steps 6, 9, 10, and 12. Particularly, the bounds are given by $\nu_{2_{\mathrm{LB}}}=[\boldsymbol{\Lambda}]_{1,1}^{2}$ and $\nu_{2_{\mathrm{UB}}}=[\boldsymbol{\Lambda}]_{1,1}^{2}+1$ in step 6. In step 9, we need to solve (31) to find optimal $\nu_{2}^{*}$ for $R>R_{\mathrm{th}}$. This $\nu_{2}^{*}$ value will then be used in step 10 to

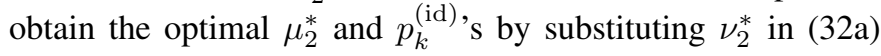
and (32b). Lastly, we need to set $\rho^{*}=1$ in step 12 .

2) Complexity Analysis: Suppose that we want to calculate $\rho^{*}$ and $\nu^{*}$ of $\mathcal{O P} 1$ or $\nu_{2}^{*}$ of $\mathcal{O P} 2$ through Algorithm 2 so as to be close up to an acceptable tolerance $\xi \ll 1$ to their globally optimal solutions. As seen from Algorithm 2, the search space interval after each GSS iteration reduces by a factor of 0.618 [34, Chap. 2.5]. This value combined with the unity maximum search length for $\rho^{*}$ and $\nu^{*}$ gives the number of iterations $c^{*}=\left\lceil\frac{\ln (\xi)}{\ln (0.618)}\right\rceil+1$ that are required to ensure that the numerical error is less than $\xi$. For example, $\xi=10^{-3}$ results in $c^{*}=16$. Note that $c^{*}$ is a logarithmic function of $\xi$ and is independent of $N_{T}, N_{R}$, and $r$. As each computation in GSS iteration for finding $\rho^{*}$ involves an inner GSS for computing $\nu^{*}$, which is repeated for at most $r_{w}$ runs, the total number of iterations required for finding the globally optimal solution of $\mathcal{O P} 1$ within an acceptable tolerance $\xi$ is given by $c_{1}^{*} \leq r_{w} c^{*}\left(c^{*}+1\right)$. Since the number of function computations in GSS is one more than the number of iterations and $r_{s} \leq r_{w} \leq r$ from Lemma 3, the total number of computations involved in solving $\mathcal{O P} 1$ are bounded by the value $r\left(\left[\frac{\ln (\xi)}{\ln (0.618)}\right]+2\right)^{2}$. Hence, the computational complexity of Algorithm 2 is $\mathcal{O}(r)$, i.e., linear in $r$. This complexity witnesses the significance of Algorithm 2 over Algorithm 1. Instead of directly implementing commercial numerical solvers or subgradient methods [33] for Algorithm 1, we use the 2-D GSS method as outlined in Algorithm 2.

Regarding the required number of iterations $c_{2}^{*}$ for finding the solution of $\mathcal{O P} 2$ it must hold $c_{2}^{*} \leq r_{w} c^{*} \leq r c^{*}$. As a result, the computational complexity of the modified Algorithm 2 for $\mathcal{O P} 2$ is $\mathcal{O}\left(r\left(\left[\frac{\ln (\xi)}{\ln (0.618)}\right]+1\right)\right)=\mathcal{O}(r)$.

3) High SNR Approximation: Recalling Remark 4 in Section V-B2 holding for high SNR values and focusing on equations (26) and (27) for $R>R_{\mathrm{th}}$, it becomes apparent that, since $p_{k}^{(\mathrm{id}, \mathrm{a})}>0 \forall k=1,2, \ldots, r$, then even with the implicit consideration of (C3) one does not need to repeatedly solve the 1-D GSS over $\nu_{2}^{*}$. Thus, we only need to find $\beta \in(0,1)$ from the following equation using the 1-D GSS method:

$$
\frac{P_{T}}{2^{\frac{R}{r}} \sigma^{2}}\left(\prod_{k=1}^{r} \frac{\beta[\mathbf{\Lambda}]_{k, k}^{2}}{\beta+[\boldsymbol{\Lambda}]_{1,1}^{2}-[\mathbf{\Lambda}]_{k, k}^{2}}\right)^{\frac{1}{r}}=\sum_{k=1}^{r}\left(\frac{\beta}{\beta+[\mathbf{\Lambda}]_{1,1}^{2}-[\mathbf{\Lambda}]_{k, k}^{2}}\right) .
$$

The computational complexity of finding the globally optimal solution of $\mathcal{O P} 2$ for high $\mathrm{SNR}$ values is therefore $\mathcal{O}\left(\left\lceil\frac{\ln (\xi)}{\ln (0.618)}\right\rceil+1\right)=\mathcal{O}(1)$, i.e., independent of $r$.

\section{NUMERICAL RESULTS AND DISCUSSION}

In this section, we numerically evaluate the performance of the proposed joint TX precoding and RX UPS splitting design, and investigate the impact of various system parameters on its achievable rate-energy trade off. Unless otherwise stated, we set $\sigma^{2}=\{-100,-70\} \mathrm{dBm}$ by considering noise spectral density of $-175 \mathrm{dBm} / \mathrm{Hz}$ as well as $P_{T}=10 \mathrm{~W}$, and $\xi=10^{-4}$. Furthermore, we model $\mathbf{H}$ as $\mathbf{H}=\left\{\theta h_{i j} \mid 1 \leq i, j \leq N\right\}$ with $N \triangleq N_{R}=N_{T}=\{2,4\}$, where $\theta=\{0.1,0.05\}$ models the distance dependent propagation losses and $h_{i j}$ 's are ZMCSCG random variables with unit variance. With this definition, the average channel power gain is given by $\theta^{2}=a d^{-n}$, where $a$ is the propagation loss constant, $n$ is the path loss exponent, and $d$ is the TX-to-RX distance. So, for $a=0.1$ and $n=2$, $\theta=0.1$ represents that $d=3.16 \mathrm{~m}$. Whereas this separation becomes twice, i.e., $d=6.32 \mathrm{~m}$, for $\theta=0.05$. We assume unit transmission block duration, thus, we use the terms 'received energy' and 'received power' interchangeably. All performance results have been generated after averaging over $10^{3}$ independent channel realizations. For obtaining $\mathbf{S}^{*}$ and $\rho^{*}$ with the proposed design we have simulated Algorithm 2.

We consider $2 \times 2$ and $4 \times 4$ MIMO systems in Fig. 4 with both ideal and UPS reception and illustrate the rate-energy trade off for our proposed designs for different values for the propagation losses and noise variance parameters. 


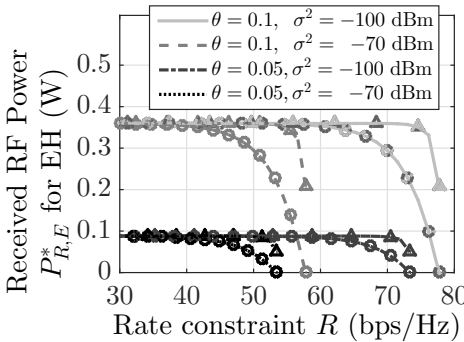

Rate constraint $R(\mathrm{bps} / \mathrm{H} z)$
(a) $2 \times 2$ MIMO system.

Fig. 4. Variation of the rate-energy trade off for varying parameters.

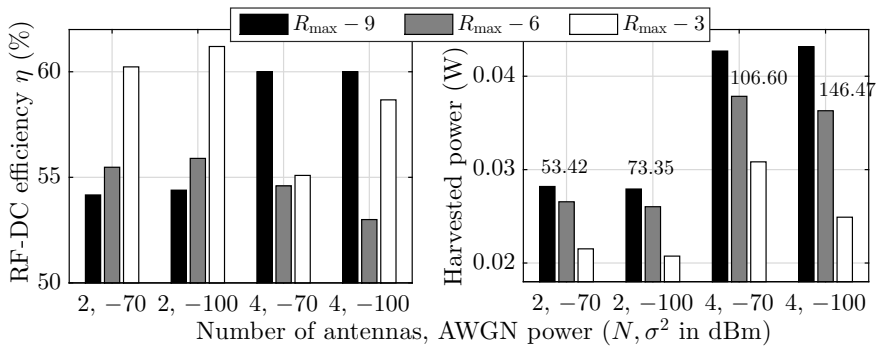

(a) Variation of rectification efficeincy

(b) Variation of harvested power $P_{H}$

Fig. 5. Variation of $\eta$ and $P_{H}$ for Powercast RF EH circuit [24] with rate $R$ near $R_{\max }$ for $P_{T}=10 \mathrm{~W} \theta=0.05$, and different values for $N$ and $\sigma^{2}$. Also, $R_{\max }$ is mentioned over the bars in (b).

As expected, our solution for $\mathcal{O P} 2$ with ideal reception outperforms that of $\mathcal{O P} 1$ that considers practical UPS reception. It is also obvious that increasing $N$ improves the rate-energy trade off. This happens because both beamforming and multiplexing gains improve as $N$ gets larger. Lesser noisy systems, when $\sigma^{2}$ decreases, and better channel conditions with increasing $\theta$ result in better trade off and enable higher achievable rates. The maximum achievable rate $R_{\max }$ in $\mathrm{bps} / \mathrm{Hz}$ for the considered four cases $\left(\theta, \sigma^{2}\right)=$ $\{(0.05,-70 \mathrm{dBm}),(0.1,-70 \mathrm{dBm}),(0.05,-100 \mathrm{dBm}),(0.1$, $-100 \mathrm{dBm})\}$ is given by $\{53.42,57.77,73.35,77.7\}$ for $N=2$ and $\{106.60,114.42,146.47,154.28\}$ for $N=4$. In addition, the average value of $R_{\mathrm{th}}$ in $\mathrm{bps} / \mathrm{Hz}$ for these cases is given by $\{17.27,18.72,26.02,27.98\}$ for $N=2$ and $\{17.46,19.05,26.92,28.73\}$ for $N=4$. When $R<R_{\mathrm{th}}$, the maximum received RF power $P_{R, E}^{*}$ for $\mathrm{EH}$ is achieved with TX energy beamforming. However, as $R$ increases and becomes substantially larger than $R_{\mathrm{th}}, P_{R, E}^{*}$ decreases till reaching a minimum value. For the latter cases, TX spatial multiplexing is adopted to achieve $R$ and any remaining received power is used for EH. Further, with $\theta$ decreasing as $\{0.1,0.01,0.001\}$, the corresponding $R_{\max }$ varies as $\{77.70,64.06,50.76\} \mathrm{bps} / \mathrm{Hz}$ for $N=2$ and $\{154.28,127.89,101.33\} \mathrm{bps} / \mathrm{Hz}$ for $N=4$. Whereas, the corresponding $P_{R, E}^{*}$ for EH with $R=0 \mathrm{bps} / \mathrm{Hz}$ varies as $\{0.36 \mathrm{~W}, 3.52 \mathrm{~mW}, 35.74 \mu \mathrm{W}\}$ for $N=2$ and $\{0.93 \mathrm{~W}$, $10.09 \mathrm{~mW}, 97.22 \mu \mathrm{W}\}$ for $N=4$ MIMO SWIPT systems.

Now considering Powercast RF EH circuit [24], we investigate the impact of $\eta$ on $P_{H}$ with varying rate requirements close to $R_{\max }$ because in this regime the corresponding $P_{R, E}^{*}$ decreases sharply as shown in Fig. 4. For each of the four cases

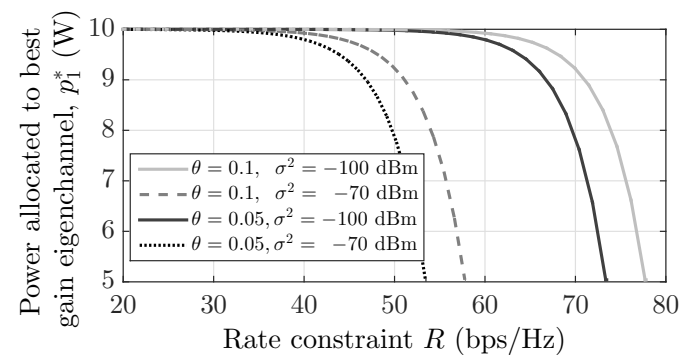

Fig. 6. Variation of the optimal power allocation $p_{1}^{*}$ of the best gain eigenchannel of a $2 \times 2$ MIMO system as a function of rate $R$.

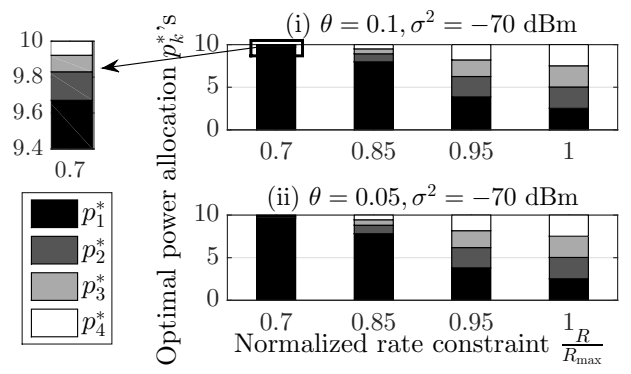

Fig. 7. Variation of optimal power allocation in a $4 \times 4$ system as a function of normalized rate $\frac{R}{R_{\max }}$ for $P_{T}=10 \mathrm{~W}, \sigma^{2}=-70 \mathrm{dBm}$.

of varying $N$ and $\sigma^{2}$ as plotted in Fig. 5, though $\eta$ does not follow any trend (increasing for first two cases and decreasing then increasing for the next two), $P_{H}$ is monotonically decreasing with increasing $R$ from $R=R_{\max }-9 \mathrm{bps} / \mathrm{Hz}$ to $R=R_{\max }-3 \mathrm{bps} / \mathrm{Hz}$, because this increase in rate $R$ results in a lower $P_{R, E}^{*}$. So, this monotonic trend of optimized $P_{H}$ in $P_{R, E}^{*}$ as depicted via Fig. 5 numerically corroborates the discussion with respect to the claim made in Proposition 1.

The variation of optimal power allocation with the proposed joint design for $\mathcal{O P} 1$ is depicted in Figs. 6 and 7 for $2 \times 2$ and $4 \times 4$ MIMO systems, respectively, as a function of $R$. Particularly, Fig. 6 illustrates the optimal power allocation $p_{1}^{*}$ over the best gain eigenchannel for $2 \times 2$ system, while the optimal power allocation $p_{1}^{*}, p_{2}^{*}, p_{3}^{*}$, and $p_{4}^{*}$ over the $r=4$ available eigenchannels is demonstrated in Fig. 7. As shown, $p_{1}^{*}$ monotonically decreases from $p_{1}^{*} \approx P_{T}$ (this happens for $R \leq R_{\mathrm{th}}$ where TX energy beamforming is adopted) to the equal power allocation $p_{1}^{*} \approx p_{2}^{*} \approx \frac{P_{T}}{2}$ (for large $R=R_{\max }$, TX spatial multiplexing is used). As from (13), $p_{1}^{*} \geq p_{2}^{*}$, we note that with $P_{T}=10 \mathrm{~W}$ for $N=2, p_{1}^{*} \geq 5 \mathrm{~W}$ in Fig. 6 . A similar trend is observed in Fig. 7. For the plotted normalized rate constraint range, most of $P_{T}$ is allocated to the best gain eigenchannel to perform TX energy beamforming, while the remaining power is allocated to the rest eigenchannels for meeting rate requirement $R$ with spatial multiplexing.

In Fig. 8, the optimal UPS ratio $\rho^{*}$ is plotted versus $R$ for $2 \times 2$ and $4 \times 4$ systems. It is shown that $\rho^{*}$ monotonically decreases with increasing $R$ in order to ensure that sufficient fraction of the received RF power is used for ID, thus, to satisfy the rate requirement. Lower $\sigma^{2}$, larger $N$ or equivalently $r$, and higher $\theta$ result in meeting $R$ with lower fraction $1-\rho$ of the received RF power dedicated for ID. Thus, for these 


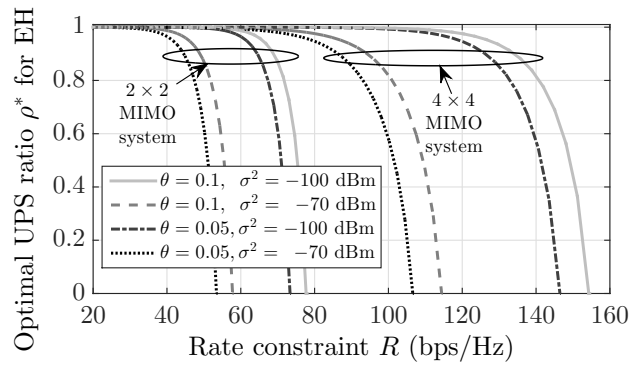

Fig. 8. Variation of the optimal UPS ratio $\rho^{*}$ for $\mathcal{O P} 1$ versus rate $R$ for $P_{T}=10 \mathrm{~W}$ and different values for $N, \theta$, and $\sigma^{2}$.

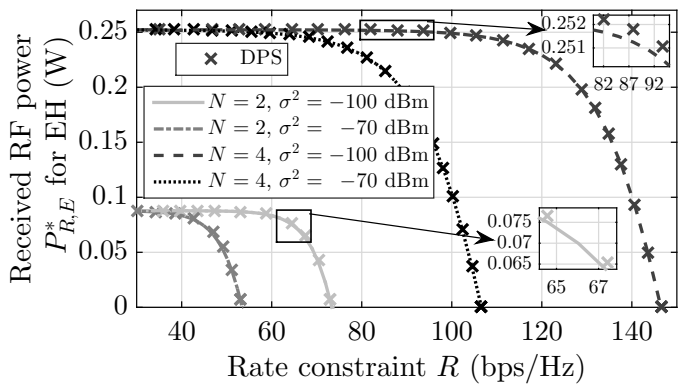

Fig. 9. Comparison against numerically optimized DPS (plotted using ' $\times$ ' markers) for $P_{T}=10 \mathrm{~W}, \theta=0.05$, and varying $N, \sigma^{2}$.

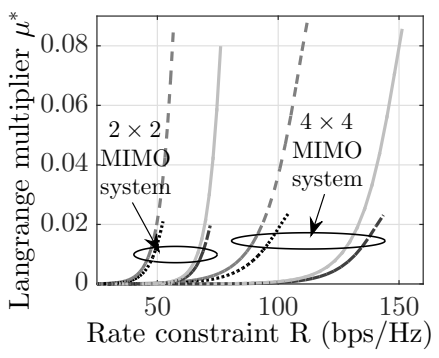

(a) Optimal Langrange multiplier $\mu^{*}$.

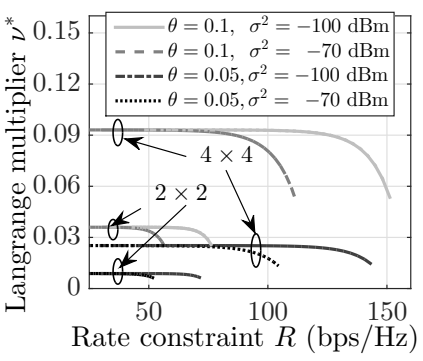

(b) Optimal Langrange multiplier $\nu^{*}$.
Fig. 10. Variation of optimal Lagrange multipliers for $\mathcal{O P} 1$ for $R>$ $R_{\mathrm{th}}, P_{T}=10 \mathrm{~W}$, and varying $N, \theta$, and $\sigma^{2}$.

cases, a larger portion of received power can be used for $\mathrm{EH}$.

We next compare the considered UPS RX operation against the more generic Dynamic PS (DPS) design, according to which each antenna has a different PS value. Since replacing DPS in our formulation results in a non-convex problem, we obtain the optimal PS ratios for the $N$ RX antennas from a $N$ dimensional linear search over the $N$ PS ratios $\rho_{1}, \rho_{2}, \ldots, \rho_{N}$ to select the best possible $N$-tuple. In Fig. 9, we plot $P_{R, E}^{*}$ for both UPS and DPS RX designs for $2 \times 2$ and $4 \times 4$ systems with $\theta=0.05$ and varying $\sigma^{2}$. In all cases, the performance of optimized DPS is closely followed by the optimized UPS with an average performance degradation of less than $0.9 \mathrm{~mW}$ for $N=2$ and $2.1 \mathrm{~mW}$ for $N=4$. This happens because the average deviation of all PS ratios in the DPS design from the UPS ratio $\rho$ is less than 0.001 . A similar observation regarding the near-optimal UPS performance was also reported in [14] for $N_{T}=1$ at TX. This study corroborates the adoption of UPS instead of DPS that incurs very high implementation complexity without yielding relatively large gains.

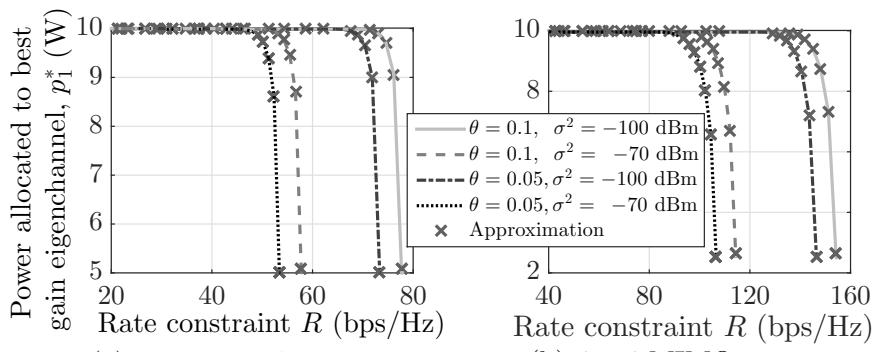

(a) $2 \times 2$ MIMO system.

(b) $4 \times 4$ MIMO system.

Fig. 11. Validating the accuracy of the proposed high SNR approximation for the globally optimal power allocation $p_{1}^{*}$ for $\mathcal{O P} 2$.

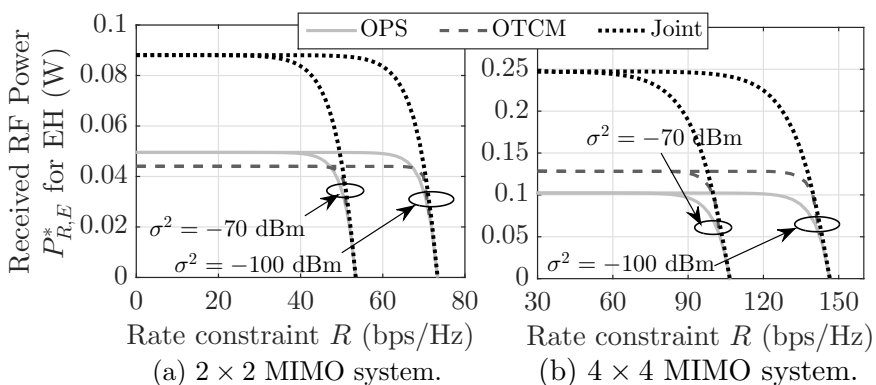

Fig. 12. Comparison of the rate-energy trade off between the proposed joint TX and RX design and the benchmark semi-adaptive schemes OPS and OTCM for $P_{T}=10 \mathrm{~W}, \theta=0.05$, and different $N$ and $\sigma^{2}$.

The Lagrange multipliers $\mu^{*}$ and $\nu^{*}$ in $\mathcal{O P} 1$ are available in closed-form as (9a) and (9b), respectively, for $R \leq R_{\mathrm{th}}$. However, one needs to solve a system of non-linear equation for these multipliers, as described in Section VI-A1, for $R>R_{\mathrm{th}}$. In Fig. 10, we plot the variation of $\mu^{*}$ and $\nu^{*}$ in $\mathcal{O P} 1$ for $R>R_{\mathrm{th}}$. As shown, $\mu^{*}$ and $\nu^{*}$ monotonically increase and decrease, respectively, with increasing $R$. The average value for $[\boldsymbol{\Lambda}]_{1,1}^{2}$ for the considered pair values $(\theta, N)=\{(0.1,2),(0.05,2),(0.1,4),(0.05,4)\}$ is $\{0.036,0.009,0.093,0.025\}$, and it is evident from Fig. 10(b) that $\nu^{*}$ is very close to its lower bound given by $\nu_{\mathrm{LB}}=$ $\rho^{*}[\boldsymbol{\Lambda}]_{1,1}^{2}$. Also, Fig. 10(a) showcases that the range of $\mu^{*}$ is similarly small to $\nu^{*}$. These findings corroborate the fast convergence of Algorithm 2 that exploits the short search space of $\nu^{*}$ in the solution of $\mathcal{O P} 1$ or $\nu_{2}^{*}$ in $\mathcal{O P} 2$. Fig. 11 includes results with the derived tight asymptotic approximation $\mathbf{S}_{\text {id,a }}^{*}$ for the globally optimal solution $\mathbf{S}_{\mathrm{id}}^{*}$ of $\mathcal{O P} 2$ in Section V-B2 using the efficient implementation of Section VI-B3. As shown, the results with TX precoding design $\mathbf{S}_{\mathrm{id,a}}^{*}\left(\right.$ or $\mathbf{P}_{\mathrm{id}, \mathrm{a}}^{*}$ ), which have been obtained from the solution of (33) in $\beta$, match very closely with the results for the globally optimal design $\mathbf{S}_{\text {id }}^{*}\left(\right.$ or $\mathbf{P}_{\text {id }}^{*}$ ) for $\mathcal{O P} 2$ implemented using Algorithm 2.

We finally present in Fig. 12 performance comparison results between the proposed joint design, as obtained from the solution of $\mathcal{O P} 1$, and two benchmark schemes. The first scheme, termed as Optimal TX Covariance Matrix (OTCM), performs optimization of $\mathbf{S}$ for a fixed UPS ratio $\rho=0.5$, and the second scheme, termed as Optimal UPS Ratio (OPS), optimizes $\rho$ for given $\mathbf{S}=\mathbf{S}_{\mathrm{WF}}$. It is observed that for 
$2 \times 2$ MIMO systems, OPS performs better than OTCM, while for $4 \times 4$ system, the converse is true. This happens because OTCM performance improves with increasing $N$ (or $r$ ). For both $N$ value, the proposed joint design provides significant energy gains over OTCM and OPS. Particularly, the performance enhancement for $N=2$ is $71.15 \%$ and $87.4 \%$, respectively, over OPS and OTCM schemes, while for $N=4$ this enhancement becomes $127.0 \%$ and $77.4 \%$, respectively.

\section{CONCLUSIONS}

In this paper, we investigated $\mathrm{EH}$ as an add-on feature in conventional MIMO systems that only requires incorporating UPS functionality at reception side. We particularly considered the problem of jointly designing TX precoding operation and UPS ratio to maximize harvested power, while ensuring that the quality-of-service requirement of the MIMO link is satisfied. By proving the generalized convexity property for a specific reformulation of the harvested power maximization problem, we derived the global jointly optimal TX precoding and RX UPS ratio design. We also presented the globally optimal TX precoding design for ideal reception. Different from recently proposed designs, the solutions of both considered optimization problems with UPS and ideal RXs unveiled that there exists a rate requirement value that determines whether the TX precoding operation is energy beamforming or information spatial multiplexing. We also presented analytical bounds for the key variables of our optimization problem formulation along with tight practically-motivated high SNR approximations for their optimal solutions. We presented an algorithm for efficiently solving the KKT conditions for the considered problem for which we designed a linear complexity implementation that is based on 2-D GSS. Its complexity was shown to be independent of the number of transceiver antennas, a fact that renders the proposed algorithm suitable for energy sustainable massive MIMO systems considered in $5 \mathrm{G}$ applications. Our detailed numerical investigation of the proposed joint TX and RX design validated the presented analysis and provided insights on the variation of the rateenergy trade off and the role of various system parameters. It was shown that our design results in nearly doubling the harvested power compared to benchmark schemes, thus enabling efficient MIMO SWIPT communication. This trend holds true for any practical non-linear RF EH model. We intend to extend our optimization framework in multiuser MIMO communication systems and consider the more general non-uniform PS reception in future works.

\section{APPENDiX A}

\section{PROOF OF LEMMA 2}

We associate the Lagrange multipliers $\nu_{2} \geq 0$ and $\mu_{2} \geq 0$ with (C2) and (C5) in $\mathcal{O P} 2$ while keeping (C3) implicit. The Lagrangian function for $\mathcal{O P} 2$ can be written as

$$
\begin{aligned}
\mathcal{L}_{2} \triangleq & P_{R}-\nu_{2}\left(\operatorname{tr}(\mathbf{S})-P_{T}\right)-\mu_{2} \\
& \times\left(R-\log _{2}\left(\operatorname{det}\left(\mathbf{I}_{N_{R}}+\sigma^{-2} \mathbf{H S H}^{\mathrm{H}}\right)\right)\right) .
\end{aligned}
$$

Let us first investigate the $R>R_{\mathrm{th}}^{\mathrm{id}}$ scenario, where for fixed $\mu_{2}>0$ and $\nu_{2}>0$, the problem of finding $\mathbf{S}$ that maximizes the Lagrangian $\mathcal{L}_{2}\left(\mathbf{S}, \mu_{2}, \nu_{2}\right)$ is expressed using (A.1) as

$\mathcal{O P} 3: \max _{\mathbf{S}} \log _{2}\left(\operatorname{det}\left(\mathbf{I}_{N_{R}}+\frac{\mathbf{H S H}^{\mathrm{H}}}{\sigma^{2}}\right)\right)-\operatorname{tr}(\mathbf{Q S})$ s.t. (C3),

where matrix $\mathbf{Q} \in \mathbb{C}^{N_{T} \times N_{T}}$ is defined as $\mathbf{Q} \triangleq$ $\frac{\ln 2}{\mu_{2}}\left(\nu_{2} \mathbf{I}_{N_{T}}-\mathbf{H}^{\mathrm{H}} \mathbf{H}\right) . \mathcal{O P} 3$ has a structure similar to the problem in [12, eq. (16)] and its bounded optimal value can be obtained for any $\mathbf{Q} \succ 0, \mu_{2} \geq 0, \nu_{2}>\lambda_{\max }\left(\mathbf{H}^{\mathrm{H}} \mathbf{H}\right)$ as

$$
\begin{aligned}
\mathbf{S}_{\mathrm{id}}^{*} & \triangleq \mathbf{Q}^{-\frac{1}{2}} \tilde{\mathbf{V}} \tilde{\boldsymbol{\Lambda}}_{\mathrm{o}} \tilde{\mathbf{V}}^{\mathrm{H}} \mathbf{Q}^{-\frac{1}{2}} \\
& =\tilde{\mathbf{V}}\left(\ln 2 \mu_{2}^{-1}\left(\nu_{2} \mathbf{I}_{r}-\boldsymbol{\Lambda}^{\mathrm{H}} \boldsymbol{\Lambda}\right)\right)^{-1} \tilde{\boldsymbol{\Lambda}}_{\mathrm{o}} \tilde{\mathbf{V}}^{\mathrm{H}},
\end{aligned}
$$

where unitary matrix $\tilde{\mathbf{V}} \in \mathbb{C}^{N_{T} \times r}$ is obtained from the reduced SVD of $\sqrt{\sigma^{-2}} \mathbf{H Q}^{-\frac{1}{2}}=\tilde{\mathbf{U}} \tilde{\mathbf{\Lambda}} \tilde{\mathbf{V}}^{\mathrm{H}}$ with unitary matrix $\tilde{\mathbf{U}} \in \mathbb{C}^{N_{R} \times r}$ and diagonal matrix $\tilde{\boldsymbol{\Lambda}} \in \mathbb{C}^{r \times r}$ containing the $r$ eigenvalues of $\sqrt{\sigma^{-2}} \mathbf{H Q}^{-\frac{1}{2}}$ in decreasing order. The entries of diagonal matrix $\tilde{\Lambda}_{\mathrm{o}} \in \mathbb{C}^{r \times r}$, obtained by using waterfilling solution [30], are related with the diagonal entries of $\tilde{\Lambda}$ as

$$
\left[\tilde{\boldsymbol{\Lambda}}_{\mathrm{o}}\right]_{i, i}=\left(1-[\tilde{\boldsymbol{\Lambda}}]_{i, i}^{-2}\right)^{+}, \forall i=1,2, \ldots r .
$$

The right-hand side of the equality in (A.2) results from rewriting $\mathbf{Q}$ as $\mathbf{Q}=\mathbf{V}\left(\frac{\ln 2}{\mu_{2}}\left(\nu_{2} \mathbf{I}_{r}-\boldsymbol{\Lambda}^{\mathrm{H}} \boldsymbol{\Lambda}\right)\right) \mathbf{V}^{\mathrm{H}}$, yielding

$$
\frac{1}{\sqrt{\sigma^{2}}} \mathbf{H Q}^{-\frac{1}{2}}=\mathbf{U} \frac{\boldsymbol{\Lambda}}{\sqrt{\sigma^{2}}}\left(\frac{\ln 2}{\mu_{2}}\left(\nu_{2} \mathbf{I}_{r}-\boldsymbol{\Lambda}^{\mathrm{H}} \boldsymbol{\Lambda}\right)\right)^{-\frac{1}{2}} \mathbf{V}^{\mathrm{H}} \text {. }
$$

Clearly, (A.4) is the reduced SVD of matrix $\sqrt{\sigma^{-2}} \mathbf{H Q}^{-\frac{1}{2}}$. Thus, we set $\tilde{\mathbf{V}}=\mathbf{V}, \tilde{\mathbf{U}}=\mathbf{U}$, and

$$
\tilde{\mathbf{\Lambda}}=\frac{\boldsymbol{\Lambda}}{\sqrt{\sigma^{2}}}\left(\frac{\ln 2}{\mu_{2}}\left(\nu_{2} \mathbf{I}_{r}-\boldsymbol{\Lambda}^{\mathrm{H}} \boldsymbol{\Lambda}\right)\right)^{-\frac{1}{2}} .
$$

Finally, $\mathbf{S}_{\text {id }}^{*}=\mathbf{F}_{\text {id }} \mathbf{F}_{\text {id }}^{\mathrm{H}}$ where $\mathbf{F}_{\text {id }} \triangleq \mathbf{V P}_{\text {id }}^{1 / 2}$ with the diagonal matrix $\mathbf{P}_{\text {id }}$ defined as $\mathbf{P}_{\text {id }} \triangleq\left(\frac{\ln 2}{\mu_{2}}\left(\nu_{2} \mathbf{I}_{r}-\boldsymbol{\Lambda}^{\mathrm{H}} \boldsymbol{\Lambda}\right)\right)^{-1} \tilde{\boldsymbol{\Lambda}}_{\mathrm{o}}$. Combining (A.3) and (A.5), the diagonal entries of $\mathbf{P}_{\text {id }}$ are

$$
p_{k}^{(\mathrm{id})}=\left(\frac{\mu_{2}^{*}}{\ln 2\left(\nu_{2}^{*}-[\boldsymbol{\Lambda}]_{k, k}^{2}\right)}-\frac{\sigma^{2}}{[\boldsymbol{\Lambda}]_{k, k}^{2}}\right)^{+},
$$

$\forall k=1,2, \ldots, r$. For $R \leq R_{\mathrm{th}}^{\mathrm{id}}, \mathbf{S}_{\mathrm{id}}^{*}=\mathbf{S}_{\mathrm{EB}}=P_{T} \mathbf{v}_{1} \mathbf{v}_{1}^{\mathrm{H}}$ is deduced from the discussion in Section IV-A. Here (C5) is satisfied at strict inequality and holds $\mu_{2}^{*}=0$ and $\nu_{2}^{*}=[\boldsymbol{\Lambda}]_{1,1}^{2}$.

\section{REFERENCES}

[1] D. Mishra and G. C. Alexandropoulos, "Harvested power maximization in QoS-constrained MIMO SWIPT with generic RF harvesting model," in Proc. IEEE CAMSAP, Curaçao, Dec. 2017, pp. 666-670.

[2] X. Lu, P. Wang, D. Niyato, D. I. Kim, and Z. Han, "Wireless networks with RF energy harvesting: A contemporary survey," IEEE Commun. Surveys Tuts., vol. 17, no. 2, pp. 757-789, Second quarter 2015.

[3] D. Mishra, S. De, S. Jana, S. Basagni, K. Chowdhury, and W. Heinzelman, "Smart RF energy harvesting communications: Challenges and opportunities," IEEE Commun. Mag., vol. 53, no. 4, pp. 70-78, Apr. 2015.

[4] I. Krikidis, S. Timotheou, S. Nikolaou, G. Zheng, D. Ng, and R. Schober, "Simultaneous wireless information and power transfer in modern communication systems," IEEE Commun. Mag., vol. 52, no. 11, pp. 104-110, Nov. 2014. 
[5] L. Xu, A. Nallanathan, and X. Song, "Joint video packet scheduling, subchannel assignment and power allocation for cognitive heterogeneous networks," IEEE Trans. Wireless Commun., vol. 16, no. 3, pp. 17031712, Mar. 2017.

[6] L. Xu, P. Wang, Q. Li, and Y. Jiang, "Call admission control with internetwork cooperation for cognitive heterogeneous networks," IEEE Trans. Wireless Commun., vol. 16, no. 3, pp. 1963-1973, Mar. 2017.

[7] L. Xu, A. Nallanathan, X. Pan, J. Yang, and W. Liao, "Security-aware resource allocation with delay constraint for noma-based cognitive radio network," IEEE Trans. Inf. Forensics Security, vol. 13, no. 2, pp. $366-$ 376, Feb. 2018

[8] L. Varshney, "Transporting information and energy simultaneously," in Proc. IEEE ISIT, Toronto, Canada, Jul. 2008, pp. 1612-1616.

[9] P. Grover and A. Sahai, "Shannon meets Tesla: Wireless information and power transfer," in Proc. IEEE ISIT, Austin, Jun. 2010, pp. 2363-2367.

[10] D. Mishra and G. C. Alexandropoulos, "Jointly optimal spatial channel assignment and power allocation for MIMO SWIPT systems," IEEE Wireless Commun. Lett., vol. 7, no. 2, pp. 214-217, Apr. 2018.

[11] X. Zhou, R. Zhang, and C. K. Ho, "Wireless information and power transfer: Architecture design and rate-energy tradeoff," IEEE Trans. Commun., vol. 61, no. 11, pp. 4754-4767, Nov. 2013.

[12] R. Zhang and C. K. Ho, "MIMO broadcasting for simultaneous wireless information and power transfer," IEEE Trans. Wireless Commun., vol. 12, no. 5, pp. 1989-2001, May 2013.

[13] D. Mishra, S. De, and C.-F. Chiasserini, "Joint optimization schemes for cooperative wireless information and power transfer over Rician channels," IEEE Trans. Commun., vol. 64, no. 2, pp. 554-571, Feb. 2016.

[14] L. Liu, R. Zhang, and K. C. Chua, "Wireless information and power transfer: A dynamic power splitting approach," IEEE Trans. Commun., vol. 61, no. 9, pp. 3990-4001, Sep. 2013.

[15] A. Nasir, X. Zhou, S. Durrani, and R. Kennedy, "Relaying protocols for wireless energy harvesting and information processing," IEEE Trans. Wireless Commun., vol. 12, no. 7, pp. 3622-3636, July 2013.

[16] S. Timotheou, I. Krikidis, S. Karachontzitis, and K. Berberidis, "Spatial domain simultaneous information and power transfer for MIMO channels," IEEE Trans. Wireless Commun., vol. 14, no. 8, pp. 4115-4128, Aug. 2015.

[17] Q. Shi, L. Liu, W. Xu, and R. Zhang, "Joint transmit beamforming and receive power splitting for MISO SWIPT systems," IEEE Trans. Wireless Commun., vol. 13, no. 6, pp. 3269-3280, Jun. 2014.

[18] Q. Shi, W. Xu, T. H. Chang, Y. Wang, and E. Song, "Joint beamforming and power splitting for MISO interference channel with SWIPT: An SOCP relaxation and decentralized algorithm," IEEE Trans. Wireless Commun., vol. 62, no. 23, pp. 6194-6208, Dec. 2014

[19] Z. Zong, H. Feng, F. R. Yu, N. Zhao, T. Yang, and B. Hu, "Optimal transceiver design for SWIPT in K-user MIMO interference channels," IEEE Trans. Wireless Commun., vol. 15, no. 1, pp. 430-445, Jan 2016.

[20] X. Li, Y. Sun, F. R. Yu, and N. Zhao, "Antenna selection and power splitting for simultaneous wireless information and power transfer in interference alignment networks," in Proc. IEEE GLOBECOM, Austin, USA, Dec 2014, pp. 2667-2672

[21] Z. Hu, C. Yuan, F. Zhu, and F. Gao, "Weighted sum transmit power minimization for full-duplex system with SWIPT and self-energy recycling," IEEE Access, vol. 4, pp. 4874-4881, Jul. 2016.

[22] K. Xiong, B. Wang, and K. J. R. Liu, "Rate-energy region of SWIPT for MIMO broadcasting under nonlinear energy harvesting model," IEEE Trans. Wireless Commun., vol. 16, no. 8, pp. 5147-5161, Aug. 2017.

[23] E. Boshkovska, R. Morsi, D. W. K. Ng, and R. Schober, "Power allocation and scheduling for SWIPT systems with non-linear energy harvesting model," in Proc. IEEE ICC, Kuala Lumpur, May 2016.

[24] Powercast. [Online]. Available: http://www.powercastco.com.

[25] D. Mishra and S. De, "Optimal relay placement in two-hop RF energy transfer," IEEE Trans. Commun., vol. 63, no. 5, pp. 1635-47, May 2015.

[26] T. Le, K. Mayaram, and T. Fiez, "Efficient far-field radio frequency energy harvesting for passively powered sensor networks," IEEE $J$. Solid-State Circuits, vol. 43, no. 5, pp. 1287-1302, May 2008.

[27] S. Boyd and L. Vandenberghe, Convex Optimization. Cambridge University Press, 2004.

[28] M. Avriel, E. Diewerth, S. Schaible, and I. Zang, Generalized Concavity. Philadelphia, PA, USA: SIAM, vol. 63, 2010.

[29] M. S. Bazaraa, H. D. Sherali, and C. M. Shetty, Nonlinear Programming. Theory and Applications. New York: John Wiley and Sons, 2006.

[30] T. Brown, P. Kyritsi, and E. De Carvalho, Practical Guide to MIMO Radio Channel: With MATLAB Examples. John Wiley \& Sons, 2012.

[31] E. Telatar, "Capacity of multi-antenna Gaussian channels," Europ. Trans. Telecommun., vol. 10, no. 6, pp. 585-595, Nov. 1999.
[32] P. He, L. Zhao, S. Zhou, and Z. Niu, "Water-filling: A geometric approach and its application to solve generalized radio resource allocation problems," IEEE Trans. Wireless Commun., vol. 12, no. 7, pp. 36373647, Jul. 2013.

[33] S. Boyd, L. Xiao, and A. Mutapcic, Subgradient methods, ser. Lecture Notes. Stanford Univ., Apr. 2003

[34] A. D. Belegundu and T. R. Chandrupatla, Optimization Concepts and Applications in Engineering. Cambridge University Press, 2011.

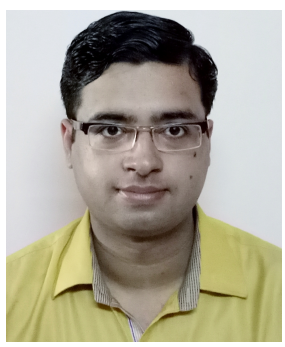

Deepak Mishra (S'13-M'17) received the B.Tech degree in Electronics and Communication Engineering from the Guru Gobind Singh Indraprastha University, Delhi, India, in 2012, and the Ph.D. degree in Electrical Engineering from the Indian Institute of Technology Delhi, India, in 2017. He is currently a Postdoctoral Researcher in the Department of Electrical Engineering (ISY), Linköping University, Linköping, Sweden. His research interests include energy harvesting cooperative communication networks, massive MIMO, physical layer security, signal processing and energy optimization schemes for uninterrupted operation of wireless networks. Dr. Mishra was selected as an Exemplary Reviewer of the IEEE TRANSACTIONS ON WIRELESS COMMUNICATIONS for 2017. He was a recipient of IBM Ph.D. Fellowship, Raman-Charpak Fellowship, and Endeavour Research Fellowship awards in 2016, 2017, and 2018, respectively.

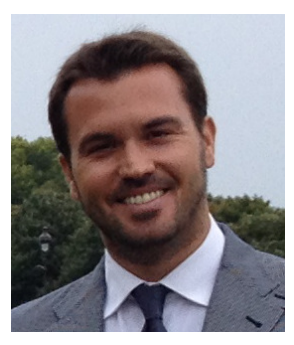

George C. Alexandropoulos (S'07-M'13-SM-17) was born in Athens, Greece, in 1980. He received the Diploma degree in computer engineering and informatics, the M.A.Sc. degree (with distinction) in signal processing and communications, and the $\mathrm{Ph} . \mathrm{D}$. degree in wireless communications from the University of Patras (UoP), Rio-Patras, Greece, in 2003, 2005, and 2010, respectively. From 2001 to 2010, he has been a Research Fellow with the Signal Processing and Communications Laboratory, Department of Computer Engineering and Informatics, School of Engineering, UoP. From 2006 to 2010, he was with the Wireless Communications Laboratory, Institute of Informatics and Telecommunications, National Center for Scientific Research Demokritos, Athens, Greece, as a Ph.D. Scholar. From 2007 to 2011, he has been collaborating with the Institute for Astronomy, Astrophysics, Space Applications, and Remote Sensing, National Observatory of Athens, Greece, where he participated in one national and two European projects. Within 2011, he also worked with the Telecommunication Systems Research Institute, Technical University of Crete, Chania, Greece, in the framework of one European project. In the summer semester of 2011, he was an Adjunct Lecturer with the Department of Telecommunications Science and Technology, University of Peloponnese, Tripoli, Greece. From 2011 to 2014, he was a Senior Researcher with the Athens Information Technology Center for Research and Education, where he has been involved with the technical management of four European projects and lectured several mathematics courses. Since 2014, he has been a Senior Researcher with the Mathematical and Algorithmic Sciences Laboratory, France Research Center, Huawei Technologies France, Boulogne-Billancourt. His research interests lie in the general areas of performance analysis and signal processing for wireless networks, with emphasis on multi-antenna systems, interference management, high-frequency communication, cooperative networking, and cognitive radios.

Dr. Alexandropoulos is a Senior Member of the IEEE Communications and Signal Processing Societies as well as a Professional Engineer of the Technical Chamber of Greece. He currently serves as an Editor for IEEE TRANSACTIONS ON WIRELESS COMMUNICATIONS and IEEE COMMUNICATIONS LETTERS. He received a Postgraduate Scholarship from the Operational Programme for Education and Initial Vocational Training II, Ministry of Education, Lifelong Learning, and Religious Affairs, Republic of Greece; a student travel grant for the 2010 IEEE Global Telecommunications Conference in Miami, USA; and the Best Ph.D. Thesis Award by a Greek University in the fields of informatics and telecommunications from the Informatics and Telematics Institute, Thessaloniki, Greece, in 2010. 